\title{
SCALED SYNTHETIC APERTURE RADAR SYSTEM DEVELOPMENT
}

\author{
A Thesis \\ presented to \\ the Faculty of California Polytechnic State University, \\ San Luis Obispo
}

\author{
In Partial Fulfillment \\ of the Requirements for the Degree \\ Master of Science in Electrical Engineering
}

by

Ryan Kristopher Green

December 2015 
(C) 2015

Ryan Kristopher Green

ALL RIGHTS RESERVED 
TITLE:

AUTHOR:

DATA SUBMITTED:

COMMITTEE CHAIR:

COMMITTEE MEMBER:

COMMITTEE MEMBER:
Scaled Synthetic Aperture Radar System Development

Ryan Kristopher Green

December 2015

John Saghri, Ph.D.

Professor of Electrical Engineering

Dean Arakaki, Ph.D.

Associate Professor of Electrical Engineering

Dennis Derickson, Ph.D.

Professor of Electrical Engineering 


\section{ABSTRACT \\ Scaled Synthetic Aperture Radar System Development \\ Ryan Kristopher Green}

Synthetic Aperture Radar (SAR) systems generate two dimensional images of a target area using RF energy as opposed to light waves used by cameras. When cloud cover or other optical obstructions prevent camera imaging over a target area, SAR can be substituted to generate high resolution images. Linear frequency modulated signals are transmitted and received while a moving imaging platform traverses a target area to develop high resolution images through modern digital signal processing (DSP) techniques.

The motivation for this joint thesis project is to design and construct a scaled SAR system to support Cal Poly radar projects. Objectives include low-cost, high resolution SAR architecture development for capturing images in desired target areas. To that end, a scaled SAR system was successfully designed, built, and tested. The current SAR system, however, does not perform azimuthal compression and range cell migration correction (image blur reduction). These functionalities can be pursued by future students joining the ongoing radar project. The SAR system includes RF modulating, demodulating, and amplifying circuitry, broadband antenna design, movement platform, LabView system control, and MATLAB signal processing. Each system block is individually described and analyzed followed by final measured data. To confirm system operation, images developed from data collected in a single target environment are presented and compared to the actual configuration. 


\section{ACKNOWLEDGMENTS}

This journey would not have been possible without the love and support of my family who provided me the opportunity to complete this degree. I would like to thank my fiancée Katie for her never-ending support, patience, and hours spent editing this document while I completed this project. I would like to thank Dr. Arakaki and Dr. Saghri for the days of time spent editing this document and their support and guidance throughout this project. Last but not least, I would like to thank my thesis partner Jason for not letting me struggle through this project alone. 


\section{TABLE OF CONTENTS}

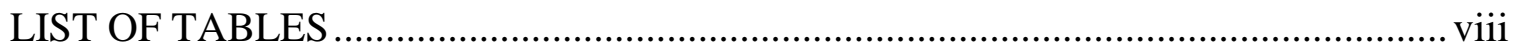

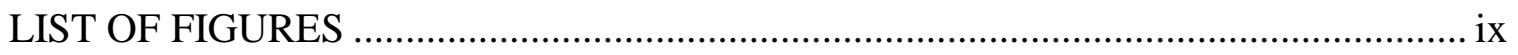

\section{CHAPTER}

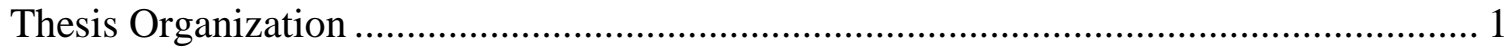

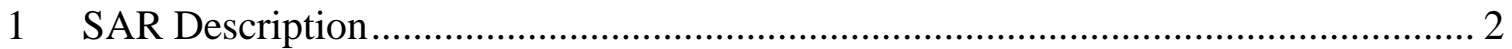

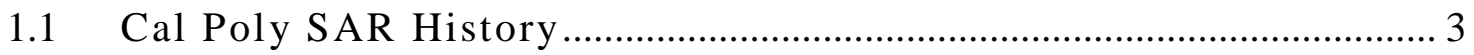

1.2 Radar Fundamentals ................................................................................. 4

1.3 Range Doppler Algorithm ..................................................................... 5

1.4 SAR IMAGE GENERATION ................................................................ 7

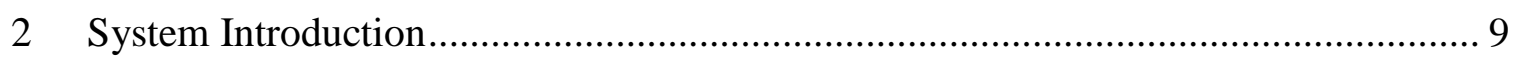

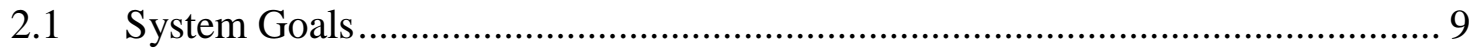

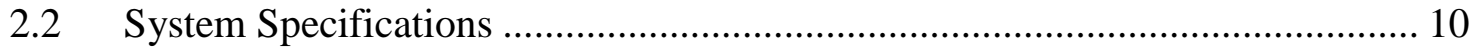

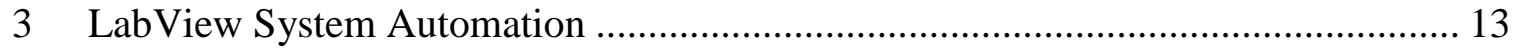

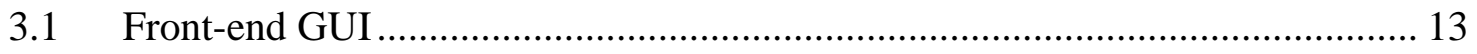

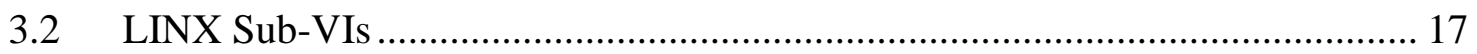

3.3 Data Collection........................................................................................... 18

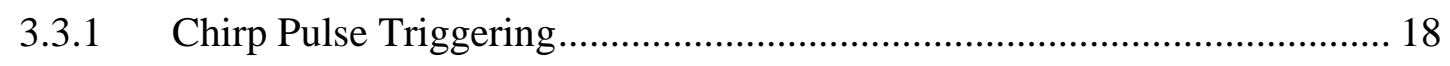

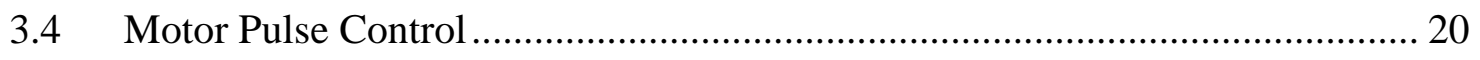

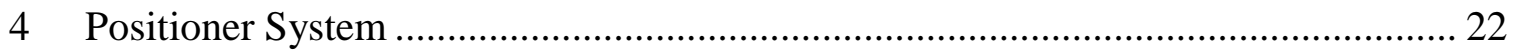

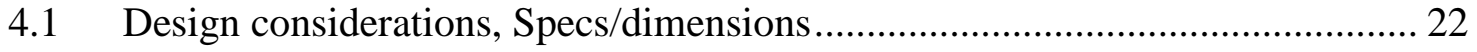

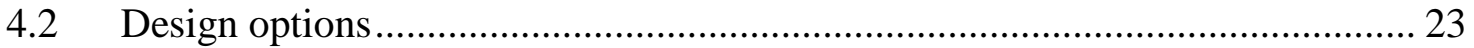

4.3 Motor and Control Circuitry ........................................................................ 27

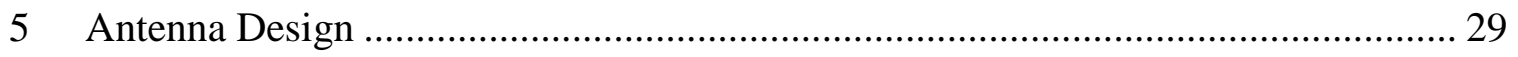

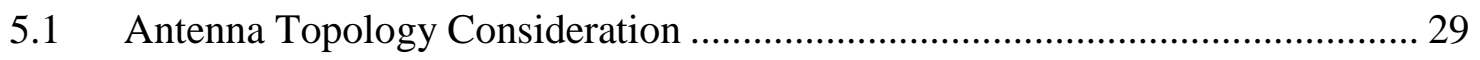

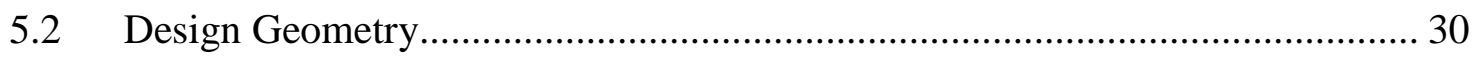

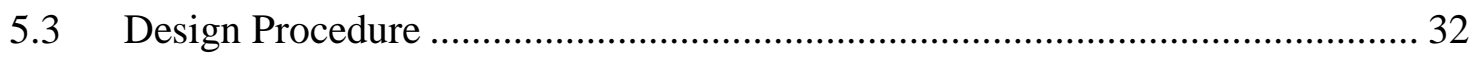

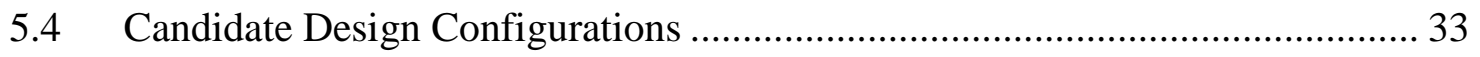

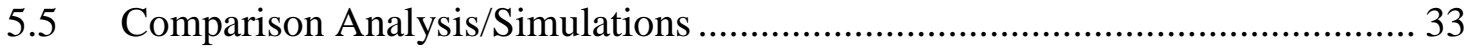

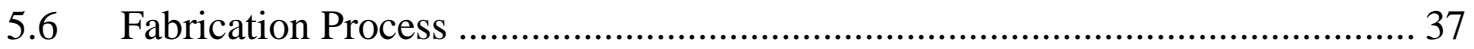

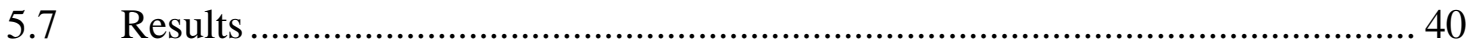

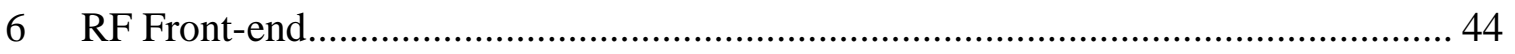

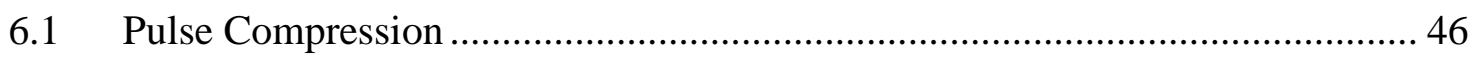




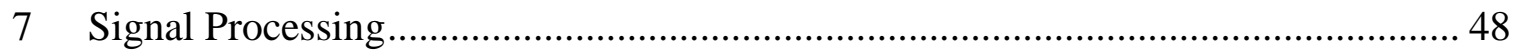

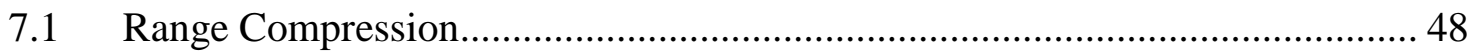

7.1.1 Hardware Range Compression ……………………............................ 50

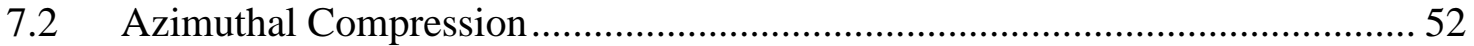

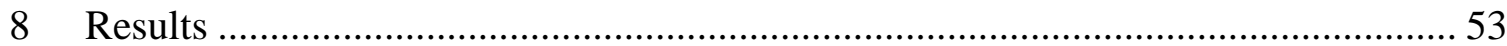

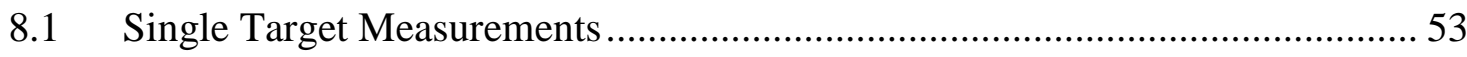

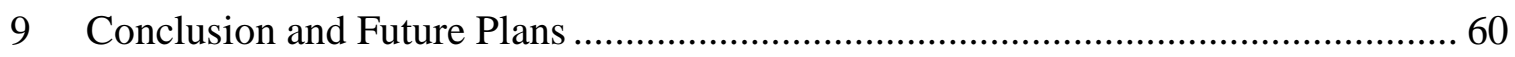

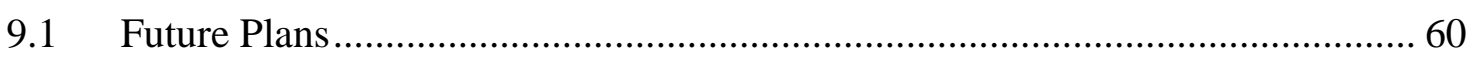

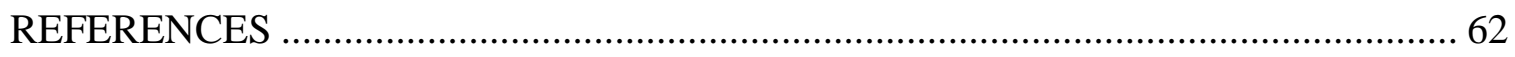




\section{LIST OF TABLES}

Table

Page

TABLE 2-1: SAR SYSTEM REQUIREMENTS

10

TABLE 5-1: ANTENNA DESIGN REQUIREMENTS; FROM MOST (1) TO

LEAST (5) IMPORTANT

TABLE 5-2: VIVALDI FLARE PERFORMANCE COMPARISON

35

TABLE 5-3: FINAL VIVALDI FLARE DESIGN GEOMETRY DIMENSIONS. 


\section{LIST OF FIGURES}

Figure

Page

FIGURE 1-1: SAR SYSTEM OPERATION; AIRPLANE TRANSMITTING

RADAR SIGNAL, IMAGING GROUND TARGET .... 2

FIGURE 1-2: SAR ATR PROJECT TOPICS AND APPLICATIONS ............................ 3

FIGURE 1-3: SIMPLE SAR IMAGE GEOMETRY ……......................................... 5

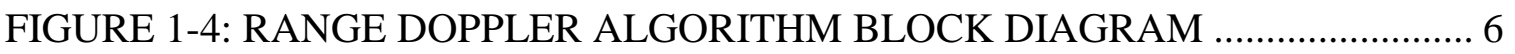

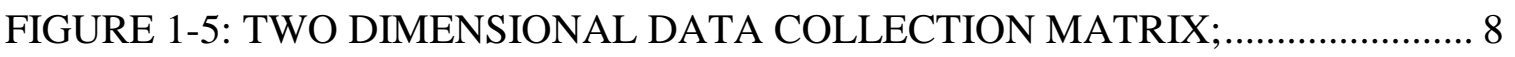

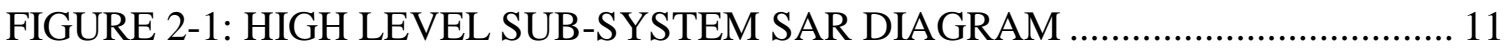

FIGURE 3-1: LABVIEW FRONT-END CONTROL PANEL, LEFT SIDE ................. 14

FIGURE 3-2: LABVIEW FRONT-END CONTROL PANEL, RIGHT SIDE............... 15

FIGURE 3-3: LABVIEW DATA COLLECTION DIAGRAM .................................... 16

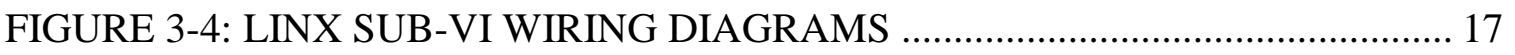

FIGURE 3-5: COLLECT DATA SUB-VI WIRING DIAGRAM ................................. 18

FIGURE 3-6: CHIRP GENERATION BLOCK DIAGRAM ….................................... 19

FIGURE 3-7: MOTOR PULSE CONTROL SUB-VI WIRING DIAGRAM................. 20

FIGURE 3-8: MOTOR PULSE CONTROL SUB-VI BLOCK DIAGRAM .................. 21

FIGURE 4-1: MOVEMENT GUIDE RAIL GEOMETRY ............................................ 23

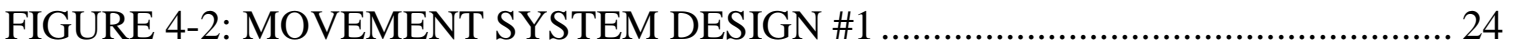

FIGURE 4-3: MOVEMENT SYSTEM DESIGN \#2 ................................................... 25

FIGURE 4-4: MOVEMENT SYSTEM FINAL DESIGN ............................................. 26

FIGURE 4-5: TI DRV8834 BREAKOUT BOARD WIRING DIAGRAM,

STEPPER MOTOR CONTROL.............................................................................28

FIGURE 5-1: VIVALDI FLARE GEOMETRY …...................................................... 30

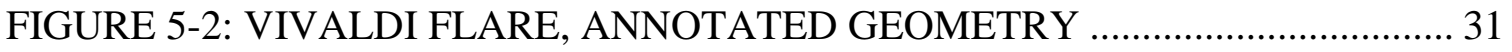

FIGURE 5-3: VIVALDI FLARE SIMULATED RETURN LOSS

COMPARISON, THREE CANDIDATE DESIGNS …...........................................35

FIGURE 5-4: SIMULATED VIVALDI FLARE ANTENNA GEOMETRY AND

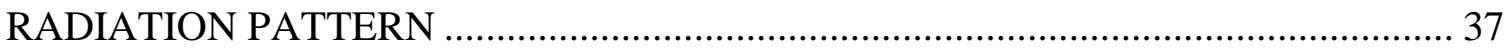

FIGURE 5-5: COMPLETED VIVALDI FLARE ANTENNA, FRONT SIDE ............... 38

FIGURE 5-6: COMPLETED VIVALDI FLARE ANTENNA, REVERSE SIDE .......... 39

FIGURE 5-7: SIMULATED VS. MEASURED RETURN LOSS COMPARISON

FOR FINAL FABRICATED VIVALDI FLARE ANTENNA PAIR .............................40

FIGURE 5-8: SIMULATED VS. MEASURED GAIN COMPARISON FOR TWO

FABRICATED VIVALDI FLARE ANTENNAS ................................................. 41

FIGURE 5-9: VIVALDI FLARE RADIATION PATTERN COORDINATE

SYSTEM: E-PLANE (PHI SCAN) AND H-PLANE (THETA SCAN) .........................42

FIGURE 5-10: MEASURED VIVALDI FLARE \#1 RADIATION PATTERN:

CO POL H PLANE (RED), CO POL E PLANE (BLUE) .........................................43

FIGURE 6-1: RF SIGNAL CHAIN BLOCK DIAGRAM .......................................... 44

FIGURE 6-2: MEASURED TRANSMITTED SIGNAL FREQUENCY

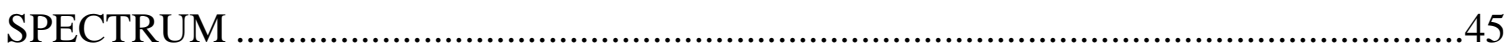

FIGURE 6-3: PULSE COMPRESSION TIMING DIAGRAM ................................. 46 
FIGURE 7-1: RANGE COMPRESSION SIGNAL PROCESSING BLOCK

DIAGRAM

FIGURE 7-2: EXAMPLE RANGE COMPRESSION RESULTS FROM

MATCHED FILTERING; RECEIVED SIGNAL MAGNITUDE LEVELS

NORMALIZED TO THE SUM OF ALL FREQUENCY COMPONENTS [6]

FIGURE 7-3: RANGE COMPRESSION RESULTS FROM PULSE

COMPRESSION AND FFT, MAGNITUDE OF RECEIVED SIGNAL LEVELS

NORMALIZED TO THE SUM OF ALL FREQUENCY COMPONENTS [6].. .51

FIGURE 8-1: TARGET SCENE GEOMETRY; SHEET TARGET 10 FT FROM

RADAR, 5 FT FROM EDGE OF RAIL MOTION

FIGURE 8-2: RAW RANGE DATA COLLECTED 5 FT ACROSS THE RAIL WITH 3X3' TARGET AT 10 FT RANGE DISTANCE.

FIGURE 8-3: RANGE DATA CALIBRATION COLLECTED WITH TX AND

RX COAX CABLES CONNECTED, ANTENNAS REMOVED .55

FIGURE 8-4: SINGLE 3'X3' COPPER SHEET TARGET LOCATED 10 FT

FROM RADAR

FIGURE 8-5: TARGET SCENE GEOMETRY FOR TARGET LOCATED 15 FT

FROM THE RADAR AND 2 FT FROM RAIL LIMIT

FIGURE 8-6: SINGLE 3'X3' COPPER SHEET TARGET LOCATED 15 FT

FROM RADAR .58 
This synthetic aperture radar thesis is a joint project with Jason Schray. Jason's thesis covers similar system components, but expands into RF component design and software which are omitted in this thesis. For a complete and thorough understanding of this project, it is advised to read both theses.

Chapter 1 includes SAR background, system description, and image generation. Chapter 2 introduces system requirements and major sub-systems: LabView, motorized platforms, antennas, RF, and signal processing. The remaining chapters, 3-5, highlight the design process for each sub-system and summarize test results compared to theory. 
Synthetic-aperture radar (SAR) employs SAR system motion relative to a target to produce a target image. Figure 1-1 illustrates general SAR system operating principles.

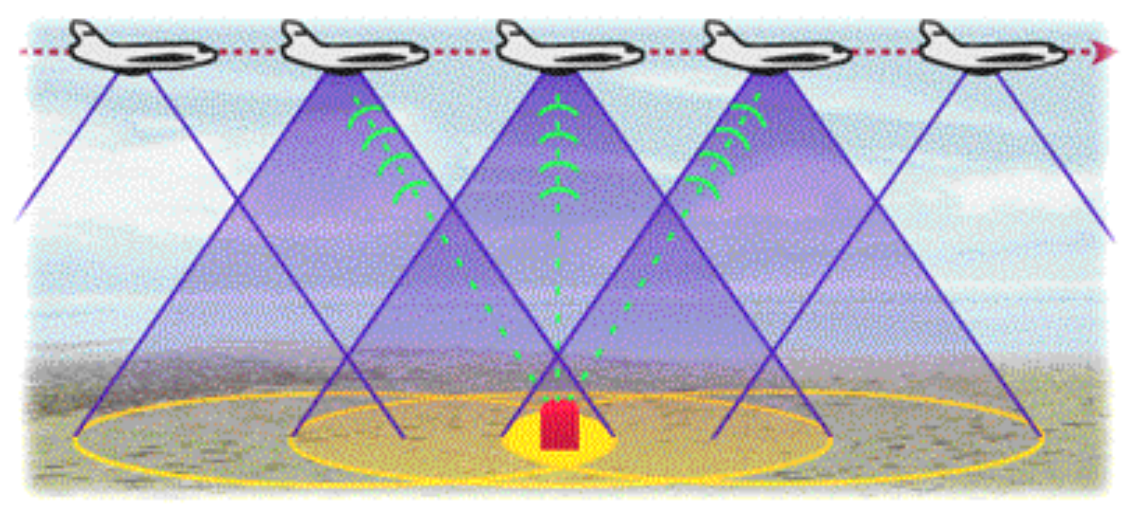

FIGURE 1-1: SAR SYSTEM OPERATION; AIRPLANE TRANSMITTING RADAR SIGNAL, IMAGING GROUND TARGET

Unlike one-dimensional systems such as police speed radars and the ever popular movie image of a white blip on a green circle in military radar screens, SAR is capable of producing high resolution target area images comparable to camera pictures. One advantage of SAR over camera captured photographs is that the radar's electro-magnetic waves can propagate through cloud cover which obscures optical images. As a result, these systems were originally developed for military surveillance purposes. These systems were housed within satellites and mounted underneath airplanes flying over enemy targets. With modern digital signal processing (DSP) hardware advances, systems can image target areas less than 100 square meters with transmit powers less than $1 \mathrm{~W}$ $(30 \mathrm{dBm})$. 


\subsection{Cal Poly SAR History}

Under the supervision of Professor John Saghri, more than two dozen Cal Poly EE graduate students (since 2004) have engaged in challenging signal processing thesis projects related to various phases of synthetic aperture radar (SAR), automatic target recognition (ATR), and target tracking as depicted in Figure 1-2 below. In the first few years, students used MSTAR (Moving and Stationary Target Acquisition and Recognition) raw data for simulation and testing; the only publicly available SAR database collected by Sandia National Laboratory in 1995. In later years, since unclassified raw SAR data were no longer made available, students resorted to designing and implementing a baseline SAR simulator to generate raw range-Doppler data required for testing and validating their refined tracking and ATR algorithms.

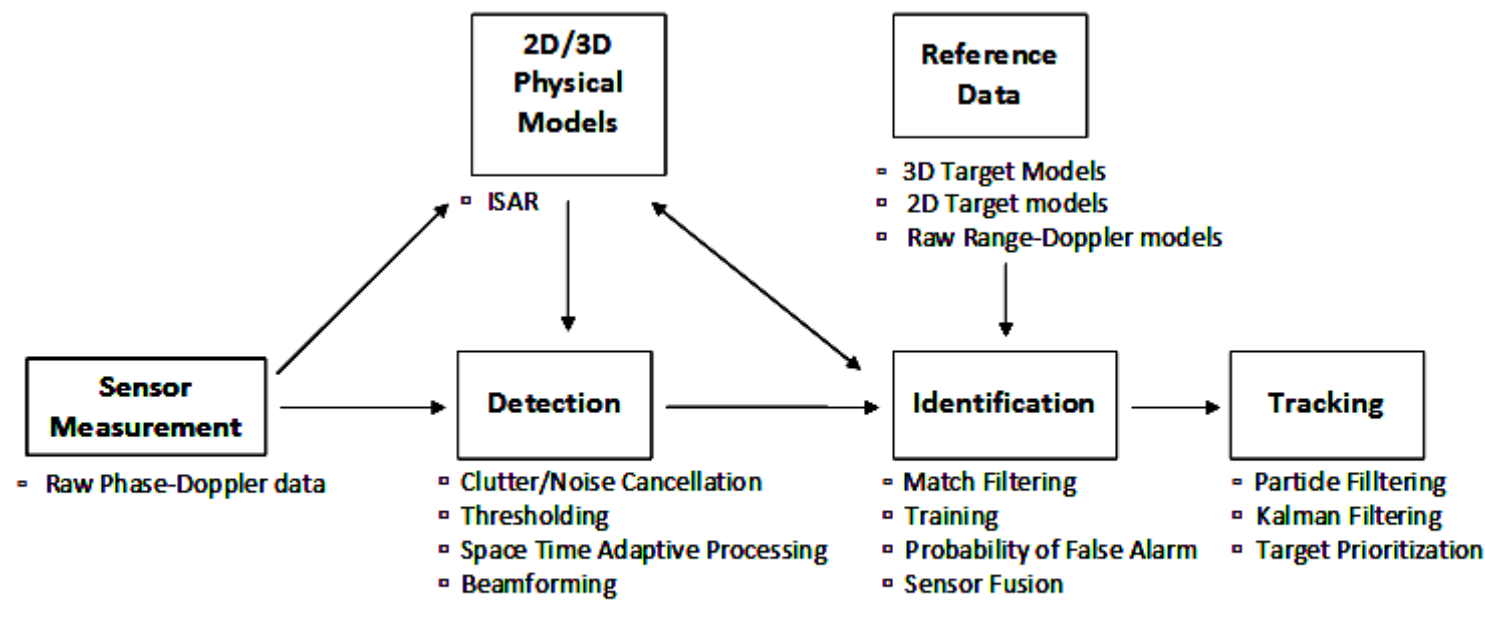

SAR ATR Signal Processing phases

FIGURE 1-2: SAR ATR PROJECT TOPICS AND APPLICATIONS

Although simulated data is useful for basic algorithm testing and validation, it cannot substitute for actual raw radar data which is inherently more complex (realistic) than simulations. The main goal of this joint thesis project has been to design and implement a 
scaled radar system within Cal Poly's anechoic chamber to collect raw radar data in support of ongoing and future students' SAR ATR projects.

\subsection{RADAR FUNDAMENTALS}

RADAR is an acronym: Radio Detection and Ranging. An RF spectrum (typically $0.1-300 \mathrm{GHz}$ ) signal is transmitted by an antenna. This signal propagates through air to an object, which reflects energy back to the antenna. Because RF spectra propagate through air at the speed of light in vacuum, $\mathrm{c}\left(\sim 3 \times 10^{8} \mathrm{~m} / \mathrm{s}\right)$, the time required for the signal to reach the target and return is directly proportional to the range-to-target distance (Range), see equation 1-1 below.

$$
\text { Range }(\text { meters })=c \frac{t}{2}
$$

where " $\mathrm{t}$ " is the total time for the signal to reach the target and return, hence divided by 2 for one flight time. The majority of ranging radar systems determine target range.

SAR systems generate images to display range to target along a platform's flight direction. 


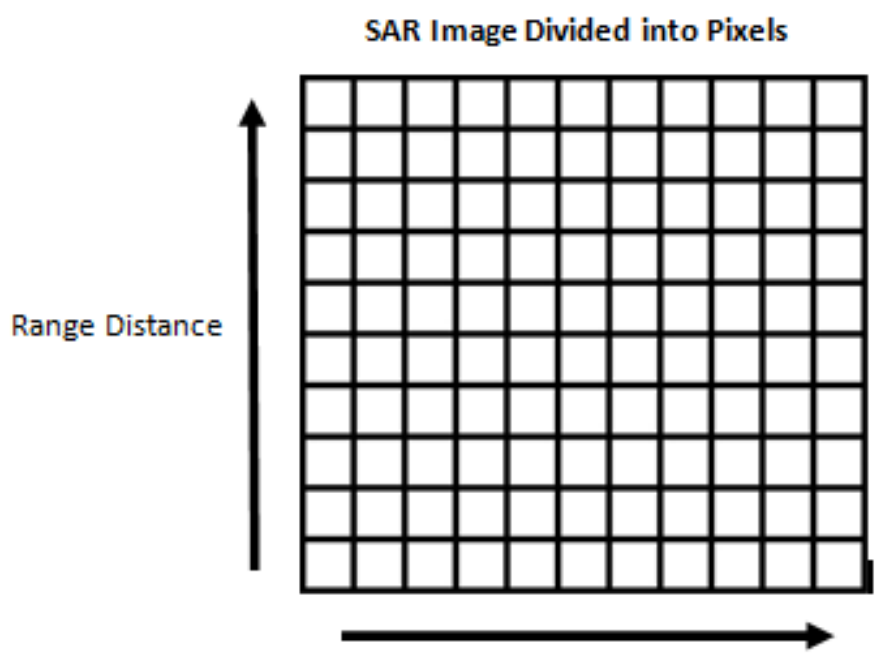

Azimuthal Distance

FIGURE 1-3: SIMPLE SAR IMAGE GEOMETRY

Target location along the platform's flight direction is its azimuthal distance along the flight path, defined in Figure 1-3 above.

Another important SAR image aspect is resolution or box size in FIGURE 1-3. SAR image resolution is divided into two parts; range and azimuthal resolution. These combine to determine SAR image cell size and therefore discernable detail in a SAR image.

\subsection{RANGE DOPPLER ALGORITHM}

Previous Cal Poly thesis projects used the Range Doppler Algorithm (RDA) to process raw data collected from a simulated SAR system into clear target images. FIGURE 1-4 below shows RDA stages used for previous thesis projects [9]. 


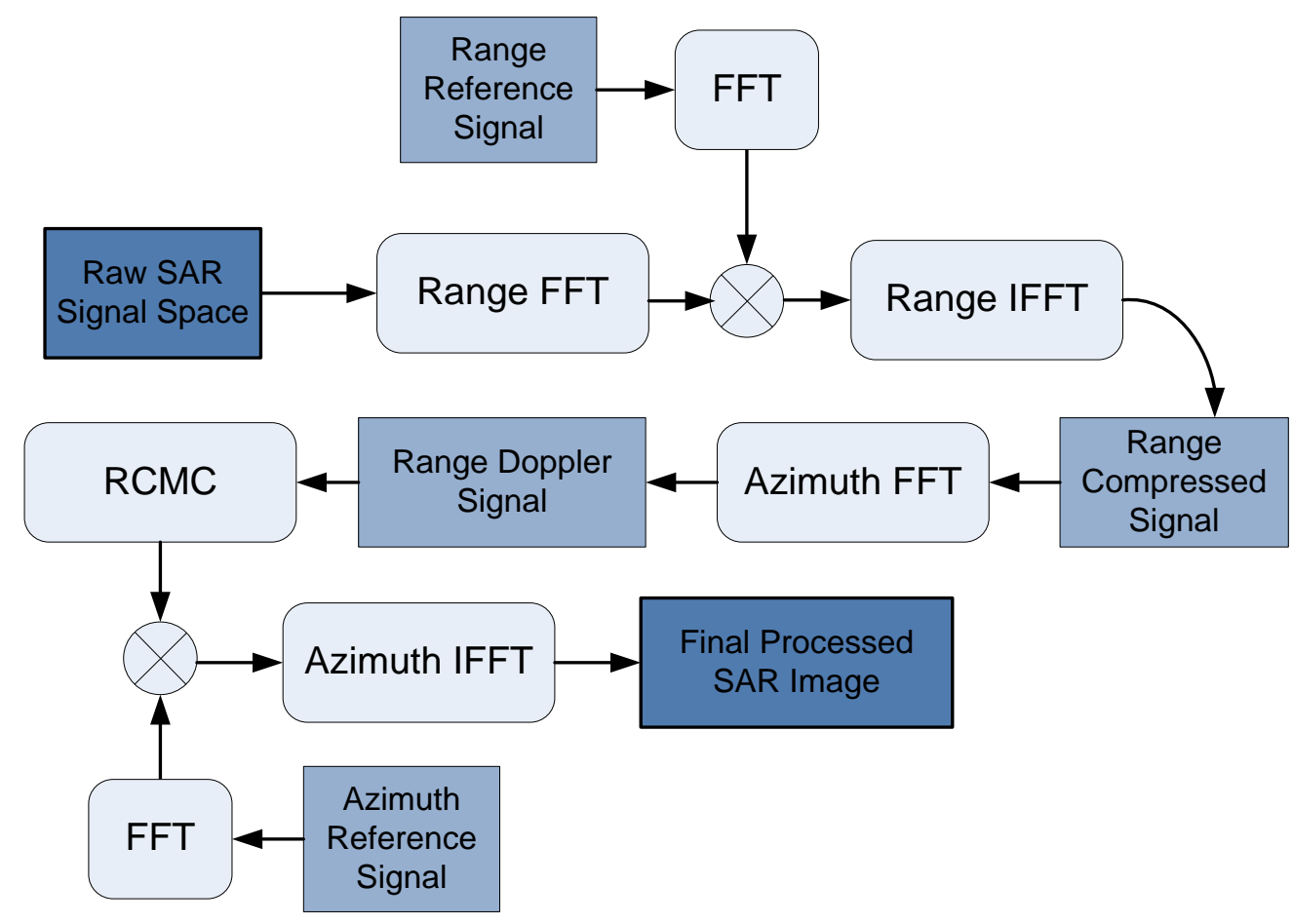

FIGURE 1-4: RANGE DOPPLER ALGORITHM BLOCK DIAGRAM

After collection and storage, raw SAR data is arranged in the format shown in FIGURE 1-3. The signal received at each azimuthal position is arranged in azimuthal bins within matrix columns. The RDA first applies the fast Fourier transform (FFT) to each azimuthal bin in the range domain. This translates the raw time domain signal into the frequency domain before filtering. Each azimuthal bin is matched filtered with the FFT of a time reversed transmit signal, which produces an amplitude peak at a frequency related to each target's range distance. After the range domain matched filtering operation, an inverse FFT returns the raw range domain data to the spatial domain. At this stage, the raw data is range compressed; the target's range location is discernable in the final image.

An FFT is applied across each azimuthal bin in the azimuthal domain. Similar to range compression, a matched filter is applied across range domain rows, defined in Figure 1-3, 
instead of azimuthal columns. The fast Fourier transformed azimuthal domain data is adjusted using range cell migration to compensate for the range compression operation. The range cell migration correction (RCMC) step corrects the inherent range domain error presented by SAR data collection. During data collection, the range-to-target changes at each location along the path of motion. As a result, the target image is "blurred" over many range bins defined by equation 1-2 below [19].

$$
\Delta R\left(f_{n}\right)=\frac{\lambda^{2} \mathrm{R}_{\mathrm{o}} f_{n}{ }^{2}}{8 \mathrm{v}_{\mathrm{p}}{ }^{2}}
$$

where $\Delta \mathrm{R}$ is the change in range to target at each azimuthal location, $\mathrm{f}_{\mathrm{n}}$ is the azimuthal frequency at each data point, $\mathrm{R}_{\mathrm{o}}$ is the minimum range to target, $\mathrm{v}_{\mathrm{p}}$ is platform velocity, and $\lambda$ is the transmitted signal wavelength. Equation 1-2 is used to correct target range by compressing target locations into correct range bins.

Finally, azimuthal compression is performed across each range bin. This process is identical to range compression, except raw azimuthal data is matched filtered with the platform's Doppler frequency spectrum. This spectrum is calculated for the platform's speed and relative range-to-target at each azimuthal location.

\subsection{SAR IMAGE GENERATION}

Once raw data is collected along the synthetic aperture or flight path, it is sent through multiple signal processing stages to generate the final image. The raw data is arranged in order of recorded position. This results in a two-dimensional matrix where columns represent slow time and rows represent fast time. Slow time is the azimuthal movement 
domain where data is collected at specific intervals. The fast time domain is reflected signal data at each azimuthal data location. Figure 1-5 illustrates the distinction.

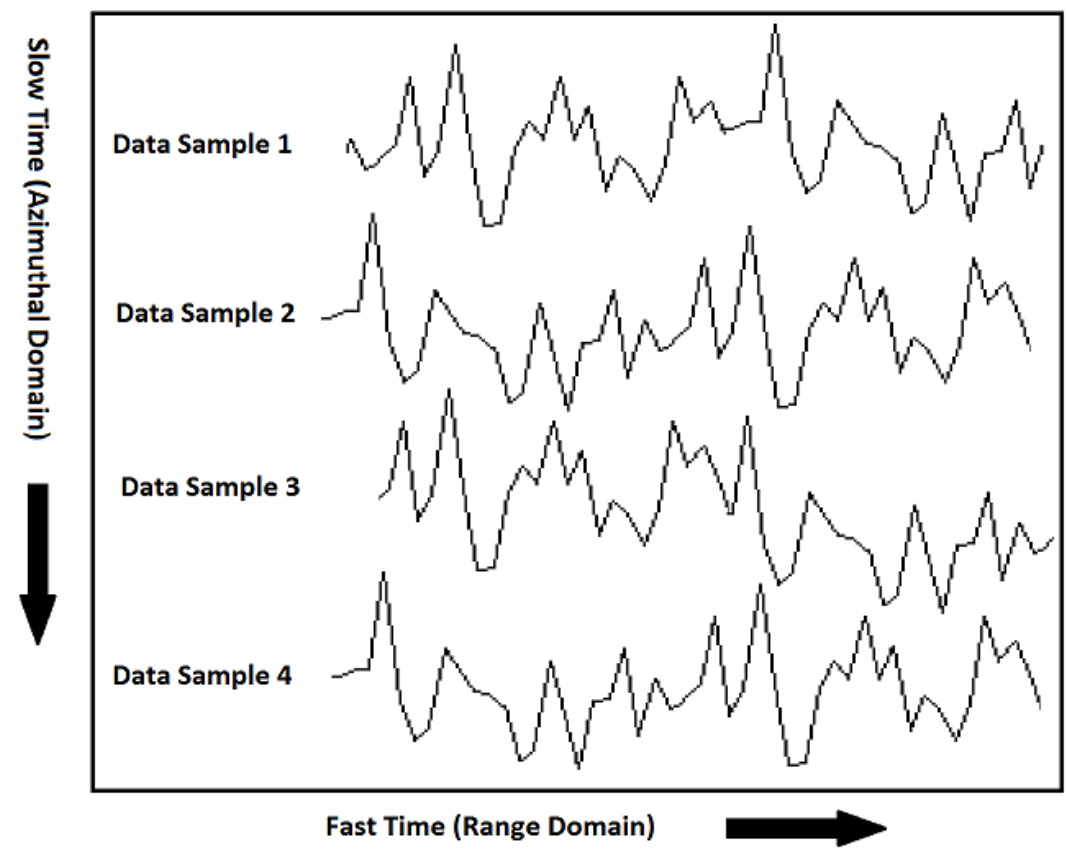

FIGURE 1-5: TWO DIMENSIONAL DATA COLLECTION MATRIX; FAST BY SLOW TIME SAMPLES

When raw data is arranged as shown in FIGURE 1-5, a blurred image can result due to varying range-to-target data across the slow time axis. Using range and azimuthal compression algorithms, the image is focused to obtain the range and azimuthal resolutions derived above. 
A typical SAR system travels on an airplane or satellite miles above the Earth's surface.

The system proposed and constructed for this thesis is a scaled version (low power, small size, transportable) of a typical SAR system. Successful short-range SAR systems [1] inspired a rail-guided moving platform for azimuthal motion relative to the target, similar to airplanes and satellites. Initially, the system was designed to operate in the Cal Poly anechoic chamber for two reasons:

1) Reduced backscatter from non-target objects; reduces noise, improves images.

2) Limit SAR system interference from nearby wireless systems such as WiFi networks, and potential cellular devices.

During system definition, it was determined that the anechoic chamber's size is insufficient for the proposed project. The size of the anechoic chamber limits the maximum transmit antenna-to-target distance to 3 meters. As described in the range resolution discussion above, decreasing target distance to 3 meters decreases time delay in the range direction to a point where the received signal power is overcome by low frequency noise due to chirp rate limitations. This problem is addressed in Section 8.1. As a result of target range limitations, it was decided to operate the SAR system outside the anechoic chamber.

\subsection{SYSTEM GOALS}

With previous thesis projects completely focused on signal processing and post processing of simulated and/or publicly released military SAR data, the focus of this thesis is primarily on recording data from an actual SAR system. Commercial SAR 
systems include advanced features to create relatively high resolution and clear images.

To build a working system in the allotted time, many advanced features (range cell migration, range gating, azimuthal compression, chirp signal linearization) are discarded to guarantee basic system functionality. Future projects may add on to this base system to improve functionality and results. The ultimate goal of this thesis is to design and implement a working SAR system capable of producing target landscape images.

\subsection{SYSTEM SPECIFICATIONS}

System specifications were selected to simplify the RF section; a $2 \mathrm{GHz}$ center frequency was chosen due to component availability and existing measurement equipment during implementation. To reduce antenna sub-system complexity, a single antenna with a duplexer was replaced with two antennas, one for transmit and one for receive. Table 2-1 summarizes system specifications and required capabilities.

TABLE 2-1: SAR SYSTEM REQUIREMENTS

\begin{tabular}{|c|c|}
\hline Center Frequency & $2 \mathrm{GHz}$ \\
\hline Chirp Pulse Bandwidth & $>1 \mathrm{GHz}$ \\
\hline Chirp Pulse Duration & $1-10 \mu \mathrm{s}$ \\
\hline $\begin{array}{c}\text { Total Azimuthal Antenna } \\
\text { Displacement }\end{array}$ & $10 \mathrm{ft}$ \\
\hline Maximum Transmit Power & $20 \mathrm{dBm}$ \\
\hline Minimum Azimuthal Resolution & $1 \mathrm{ft}$ \\
\hline Minimum Range Resolution & $<15 \mathrm{~cm}$ \\
\hline Maximum Range Distance & $50 \mathrm{ft}$ \\
\hline
\end{tabular}


Figure 2-1 illustrates the overall system, which defines major sub-systems and defines the remaining chapters.

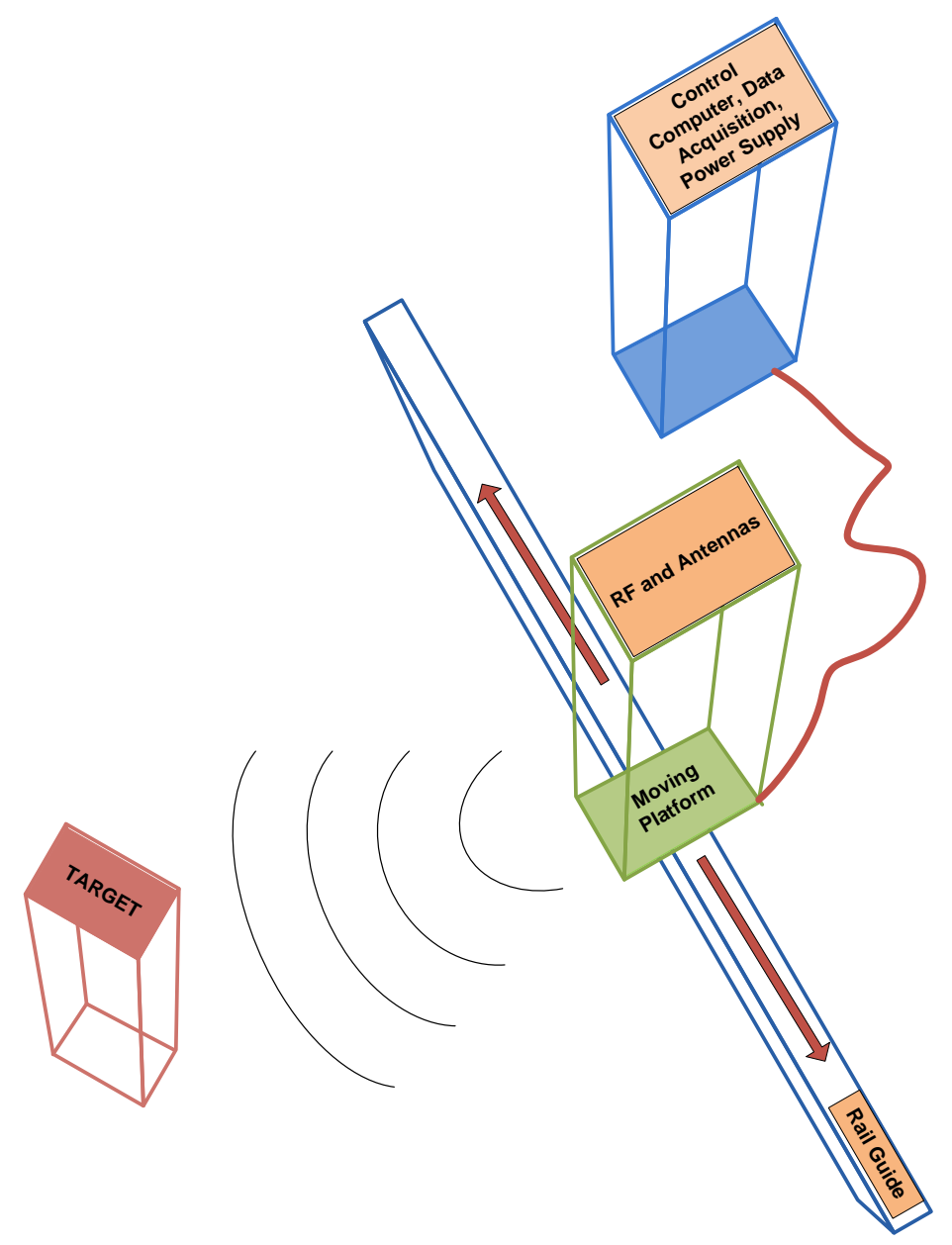

FIGURE 2-1: HIGH LEVEL SUB-SYSTEM SAR DIAGRAM

The system is divided into two sections:

1) Moving platform: carries antennas and RF sub-system

2) Control, data acquisition, and signal processing sub-systems

The two sections are connected by a $15 \mathrm{ft}$ cable bundle for biasing voltage, control, and received data acquisition signals. The control wires connect to an Arduino Mega 
contained on the moving platform which triggers chirp pulse transmission. The system control and automation is a LabView VI (virtual instrument code) that interfaces with the Arduino for pulse transmission, moves the platform to each data location, triggers the data acquisition oscilloscope, and pre-processes raw received waveforms for signal processing. 
To coordinate SAR system timing, a LabView VI was developed as a GUI (graphical user interface) to initialize system parameters and monitor data collection. Four sub-VI routines control SAR system components:

1) RF and antenna platform movement

2) Arduino micro-controller interface for chirp pulse triggering

3) Oscilloscope setup and data collection

4) Raw data pre-processing for MATLAB

\section{$3.1 \quad$ FRONT-END GUI}

The front-end GUI enables a user-specified number of recorded data points, number of pulses for averaging at each data location, and oscilloscope control parameters.

Figures 3-1 and 3-2 show the front-end control SAR system GUI. In addition to control parameters, multiple real-time data response and range-to-target estimates at each rail location are displayed. 


\section{Oscope resource name}

I/0USB0::0x0957:0x1799::MY51137451:

\section{Ramp Channel Setup Recieved Channel Setup}

Recieved Channel Name (0: Channel 1$)$

A. Channel 2 1

Recieved Channel Probe Attenuation (1.0)

1. 20

Recieved Channel Coupling (1: DC)

슨 1

Recieved Channel Vertical Range (1.0 V)

1. 20

Recieved Channel Filter Type (0: Off)

난) 0

\section{Enable Data Collection}

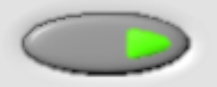

Channel to trigger from (T: Ramp, F: Received)

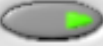

Trigger Channel Reference (1: Center)

s) Left 0

Trigger Channel Trigger Level $(0 \mathrm{~V})$

간) 1.5

Trigger Channel Edge Slope (0: Positive)

?) Positive 0

Number of Pulses to Average

각 1

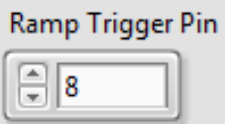

Collected Data File Path

q.......

Recieved Channel Time per Division ( 0.0005 s)

7) $5 \mathrm{E}-7$

Progress

\# of Datapoints Covered

0

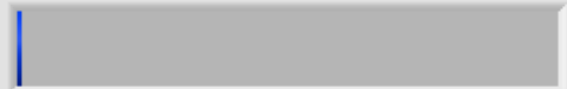

FIGURE 3-1: LABVIEW FRONT-END CONTROL PANEL, LEFT SIDE 


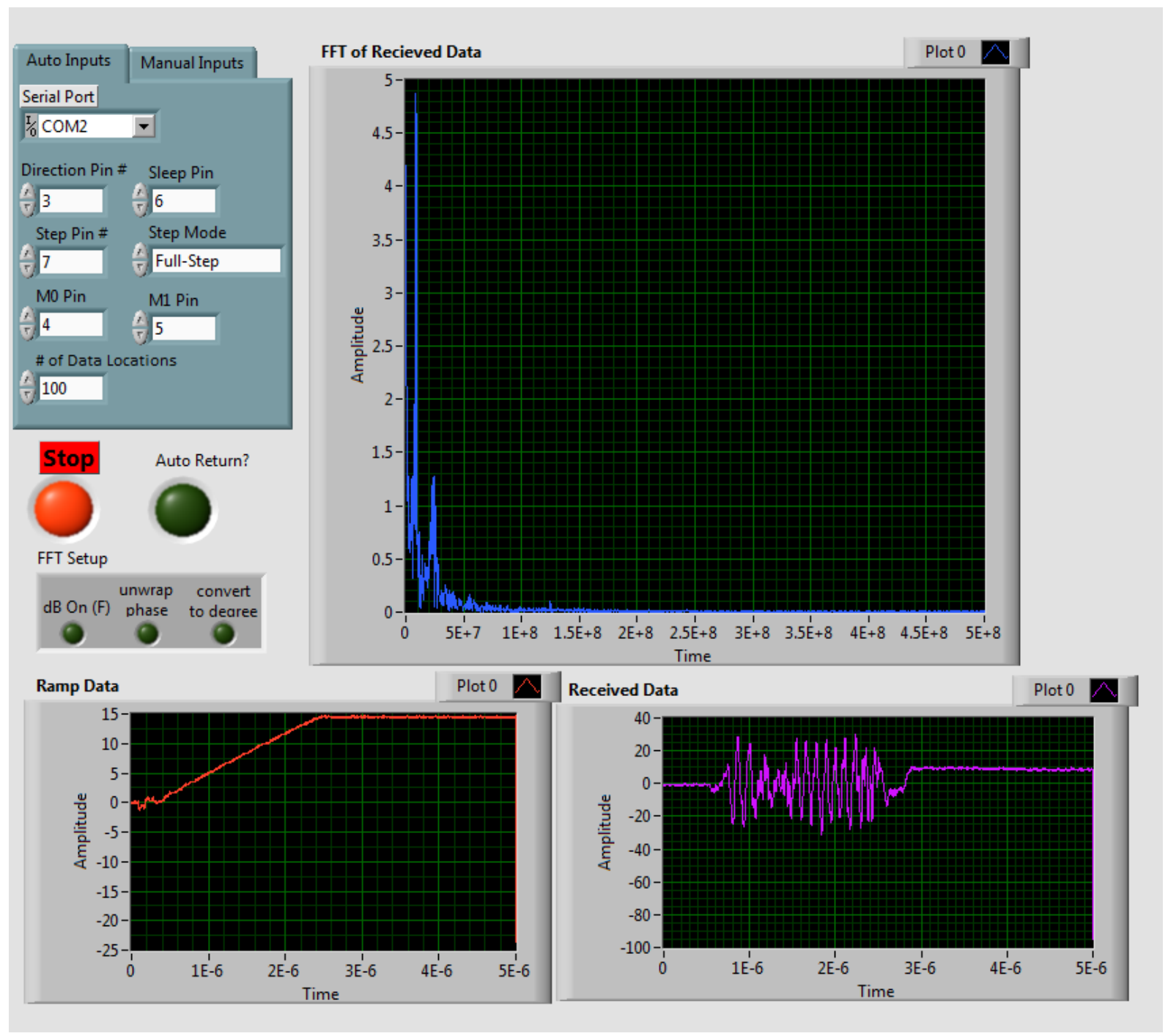

FIGURE 3-2: LABVIEW FRONT-END CONTROL PANEL, RIGHT SIDE

Once all oscilloscope triggering and motor control inputs are defined, data collection begins. The block diagram in Figure 3-3 summarizes LabView operations during data collection. 


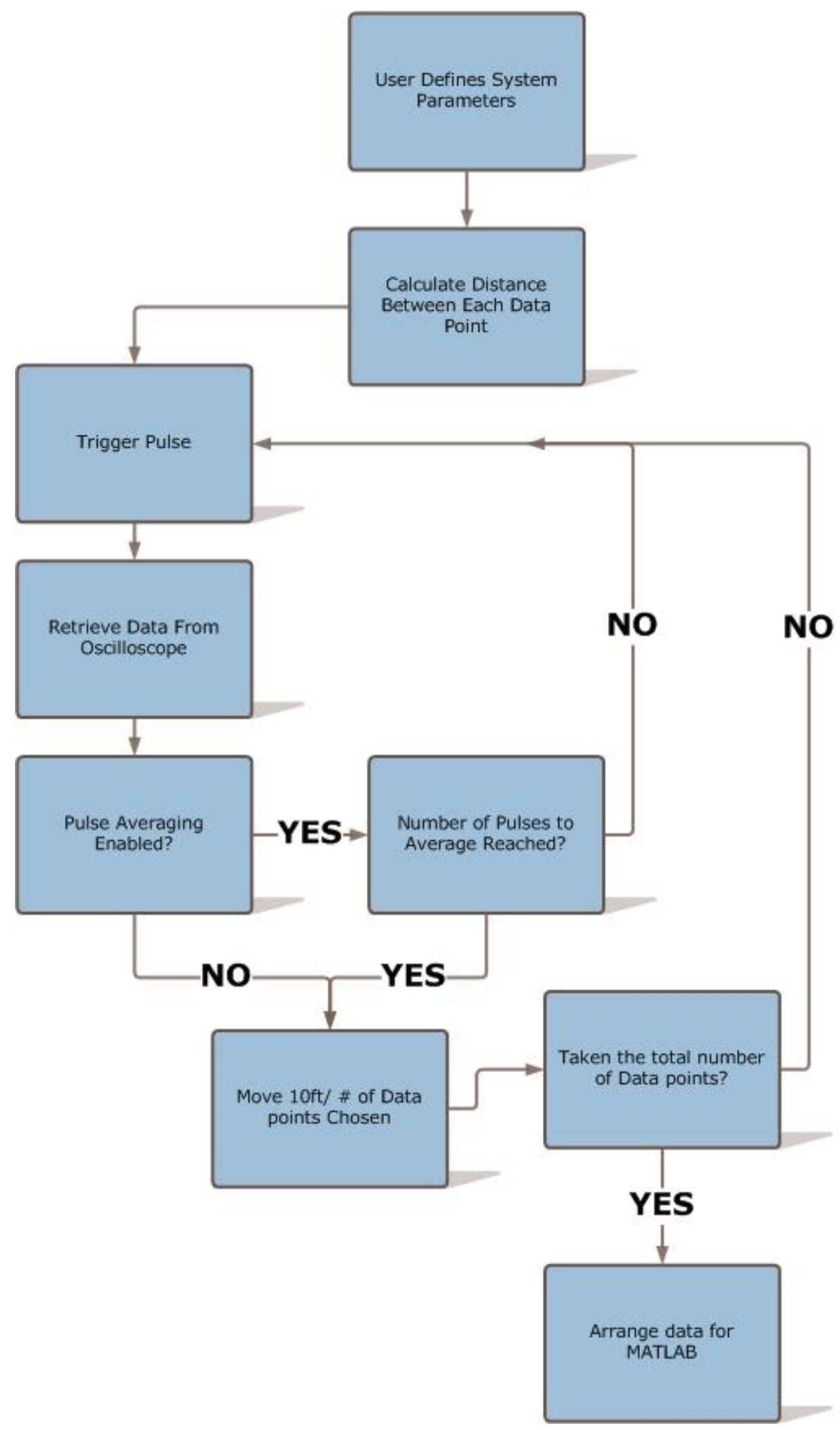

FIGURE 3-3: LABVIEW DATA COLLECTION DIAGRAM 
During data collection, a pulse is transmitted at specific intervals along the rail, determined by rail length and user-selected number of data points. Data is sampled and stored using a digital oscilloscope and sent to the computer. A "received signal average over multiple pulses" option is also available. Individual sub-VIs used to complete each data collection diagram task (Figure 3-3) are described below.

\subsection{LINX SUB-VIS}

LINX is an open source API (application program interface) for interfacing microcontrollers with LabView. It provides microcontroller-stored firmware and sub-VIs for larger projects. The sub-VIs open a COM port for microcontroller communications, digital and analog pin control, and advanced micro-controller functions. For this project, only COM port access and digital pin control microcontroller functions are required.

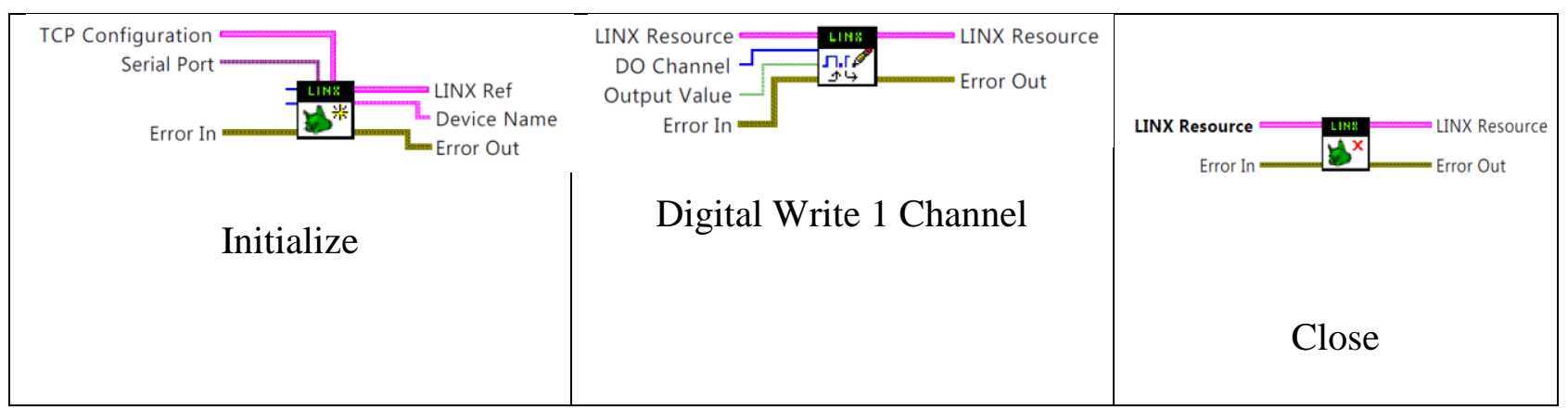

FIGURE 3-4: LINX SUB-VI WIRING DIAGRAMS

The "Initialize" VI opens the serial COM port for microcontroller communications and a LINX resource for LINX VI microcontroller communications. The "Digital Write 1 Channel" VI sets a logic value on the microcontroller digital pin defined by the "Do Channel" input. Finally, the "Close" VI closes the microcontroller serial COM port to prepare the microcontroller for the next data collection cycle without reset. 


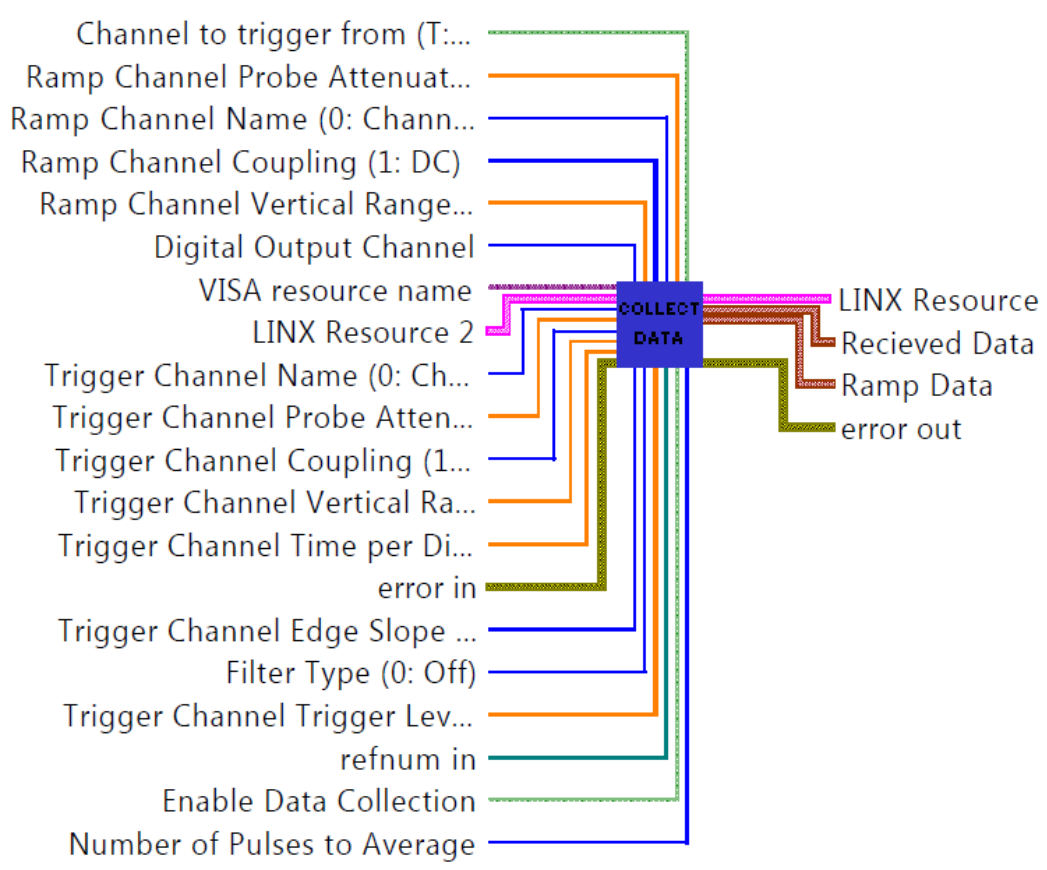

FIGURE 3-5: COLLECT DATA SUB-VI WIRING DIAGRAM

The "collect data" sub-VI controls chirp pulse transmission and oscilloscope data collection settings.

\subsubsection{CHIRP PULSE TRIGGERING}

Interfacing a computer with a micro-controller requires COM port management and data transmission protocols such as UART. Fortunately, the LINX open source project simplifies the Arduino microcontroller interface by providing pre-built LabView VIs for basic tasks such as toggling digital input and output pins. The LINX project also provides firmware for continuous microcontroller communications with LabView.

The SAR system microcontroller's primary functions are to trigger chirp pulse transmissions and to control RF and antenna system rail position via stepper motor commands. Figure 3-6 summarizes LabView and micro-controller interactions to 
generate the chirp signal. To begin chirp pulse transmission, the LabView VI toggles the voltage ramp generator circuit microcontroller pin. Upon rising edge transition, a capacitor is charged through a current mirror circuit. The voltage across the capacitor increases linearly with a constant applied current, resulting in a voltage ramp, which is applied to the VCO's Vtune pin. The ramp signal and VCO generate the $1.5-2.5 \mathrm{GHz}$ chirp signal. The microcontroller pin connected to the ramp generating circuit is reset (low state) to prepare for the next ramp.

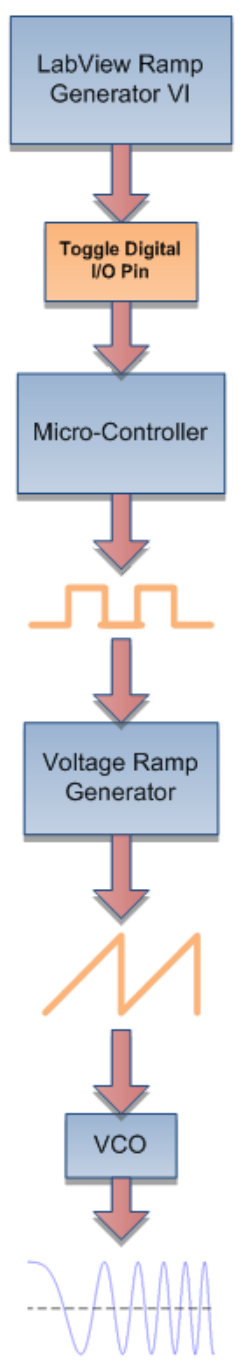

FIGURE 3-6: CHIRP GENERATION BLOCK DIAGRAM 
Shortly after pulse transmission, a target reflects the signal back to the receiving antenna. Due to the extremely short time delay (ns) between pulse transmission and reflection, the oscilloscope is set to single trigger mode to guarantee complete reflected pulse capture. The oscilloscope fills its sample memory when the user-defined trigger signal is received. The oscilloscope also records the VCO voltage ramp. Signal processing techniques accurately determine the VCO chirp rate required for image generation, as described by equation 7-4 in Chapter 7: Signal Processing.

\subsection{MOTOR PULSE CONTROL}

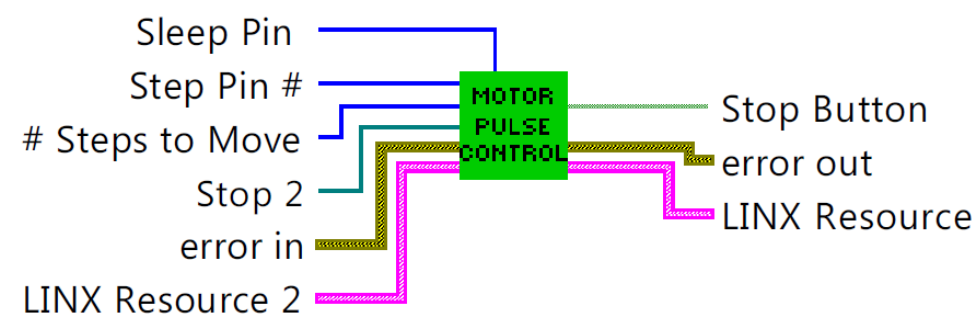

FIGURE 3-7: MOTOR PULSE CONTROL SUB-VI WIRING DIAGRAM

The motor pulse control sub-VI rotates the motor axle one step $\left(1.8^{\circ}\right)$. This sub-VI is used in a loop to produce continuous movement. The number of steps to move between each data point location is calculated in step 2 of Figure 3-3. The block diagram for this sub-VI is shown in Figure 3-8. 


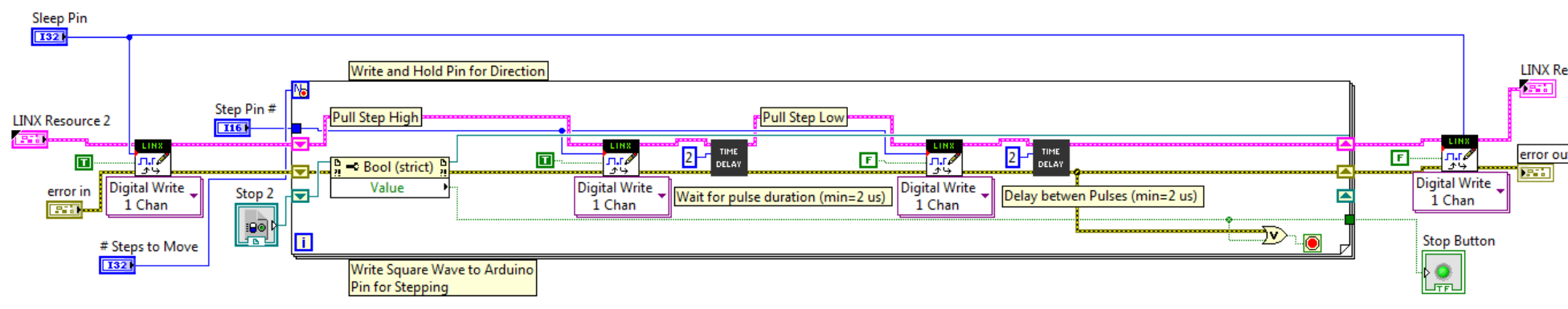

FIGURE 3-8: MOTOR PULSE CONTROL SUB-VI BLOCK DIAGRAM

An adjustable time delay between each LINX digital write command controls motor speed. In the above capture, the time between rising edges is set to $4 \mu \mathrm{s}$, which is limited by LINX to Arduino protocol delay. 
The rail positioner system provides azimuthal motion relative to a target to simulate airplane or satellite movement over the Earth. The rail system allows movement across the target scene while recording a user-defined number of data points along the azimuthal direction. The RF, antenna sub-system, and motor are installed on a rail-mounted platform. The Arduino microcontroller regulates movement along the rail through motor commands.

\subsection{DESIGN CONSIDERATIONS, SPECS/DIMENSIONS}

System performance specifications dictate rail and movement system requirements. First, the rail must be portable to allow imaging multiple target scenes - the system can be relocated by two people. Second, to limit signal reflections from the rail itself, it must be composed of non-conducting material. Though it is possible to calibrate out constant reflections from each azimuthal position's range data, initial signal processing is reduced if system interference effects are eliminated. Finally, for time efficiency, the platform must traverse the entire rail in less than a minute at top speed. This requirement reduces development time by minimizing test run times.

From the above requirements, the following specifications were developed:

1. System weight: less than 150 pounds. (Assuming one person can comfortably carry 75 pounds.)

2. System materials: plastic, wood, or other non-conducting material.

3. Minimum platform speed: 3 meters per minute. 
Before construction, it was decided to use wood for all system components due to raw material accessibility and woodworking tools.

\subsection{DESIGN OPTIONS}

Three designs were developed. All three designs follow the Figure 4-1 diagram, but differ in platform-driving motor techniques and platform-guide rail contact methods.

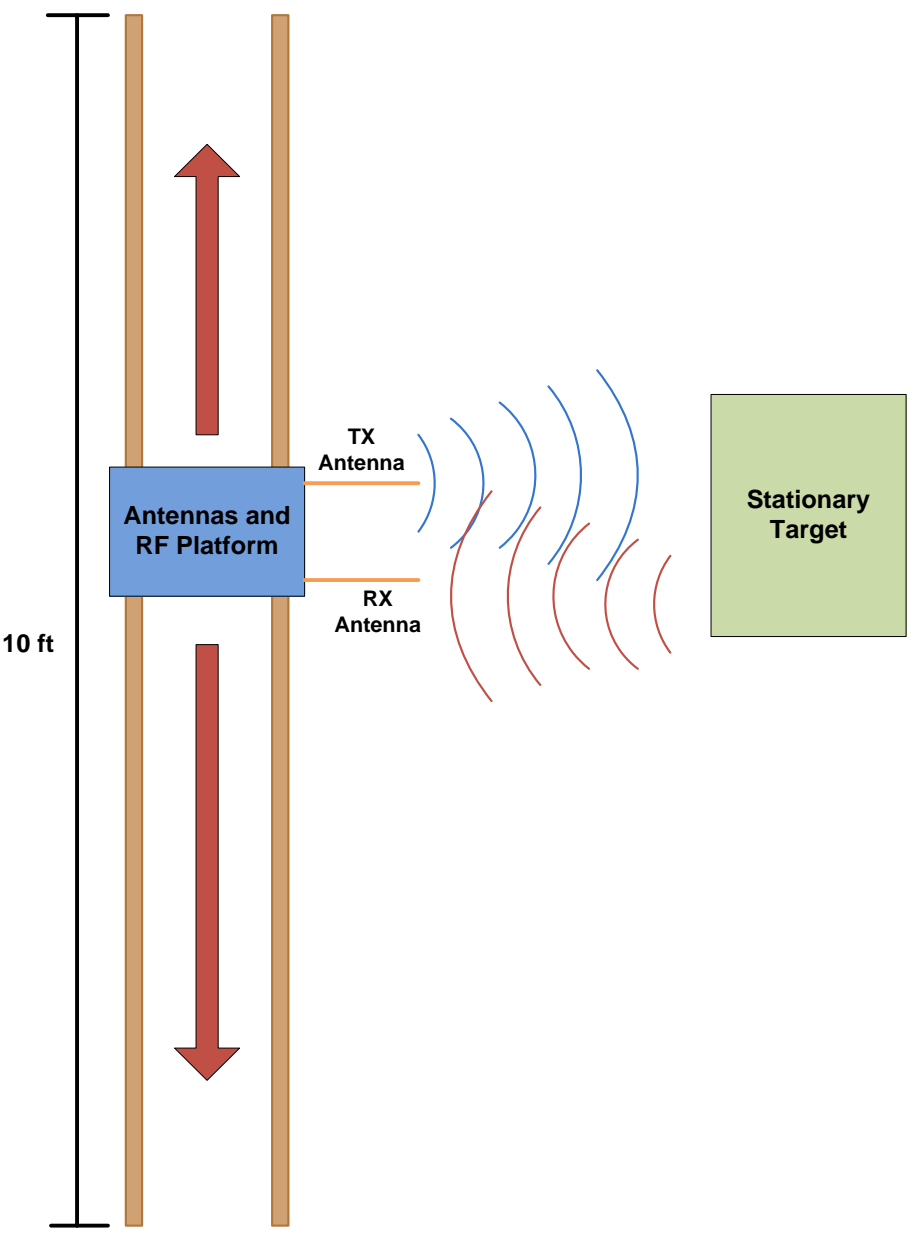

FIGURE 4-1: MOVEMENT GUIDE RAIL GEOMETRY 
The main guide rail base includes two parallel 10'x 2"x4" beams. The beams were mounted on a 3'x3' square of $3 / 4$ "' thick plywood on both ends to balance the guide rails and platform.

The first design is a variation on the rail built for scaled radar systems [1]. In this design, a screw is set parallel and centered between two wooden guide rails, see Figure 4-2. At one end, a motor connects to a threaded rod which is threaded through a nut connected to the platform. The platform's position is controlled by rotating the rod. Perspective and side views are shown below to illustrate the movement mechanism.

\section{FRONT VIEW}

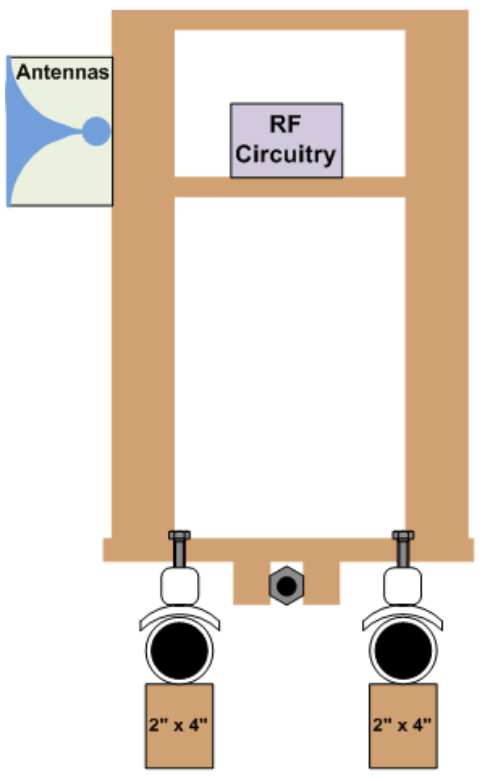

\section{SIDE VIEW}

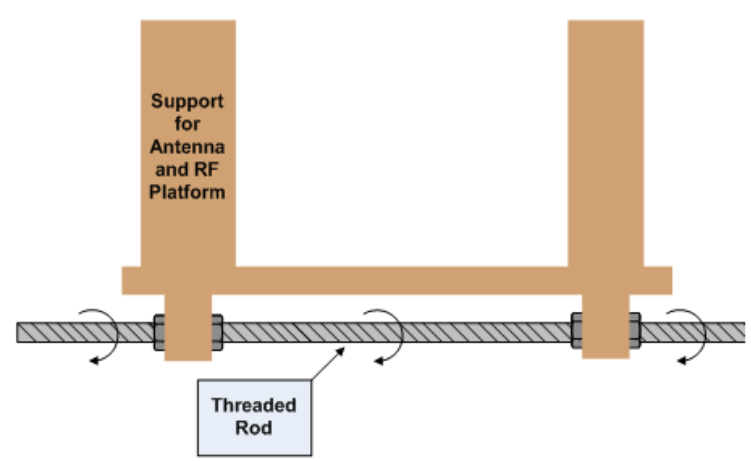

FIGURE 4-2: MOVEMENT SYSTEM DESIGN \#1

The guide rails were each outfitted with a 11/4" diameter PVC pipe. On the platform, PVC coupling sections were cut in a half circle to fit firmly over the guide rail pipes. While operable, this design had serious drawbacks. 
1. The weight of the threaded rod was too much for the motor to rotate quickly, making the platform difficult to move.

2. The screw threading was too fine and required excessive revolutions to move the platform efficiently.

3. Friction between the center platform PVC couplers and the guide rail PVC pipes slowed movement and occasionally exceeded available motor torque.

From the first design outcomes, the PVC sliding motion was replaced with the trolley wheel and rolling mechanism shown in Figure 4-3 below.

FRONT VIEW

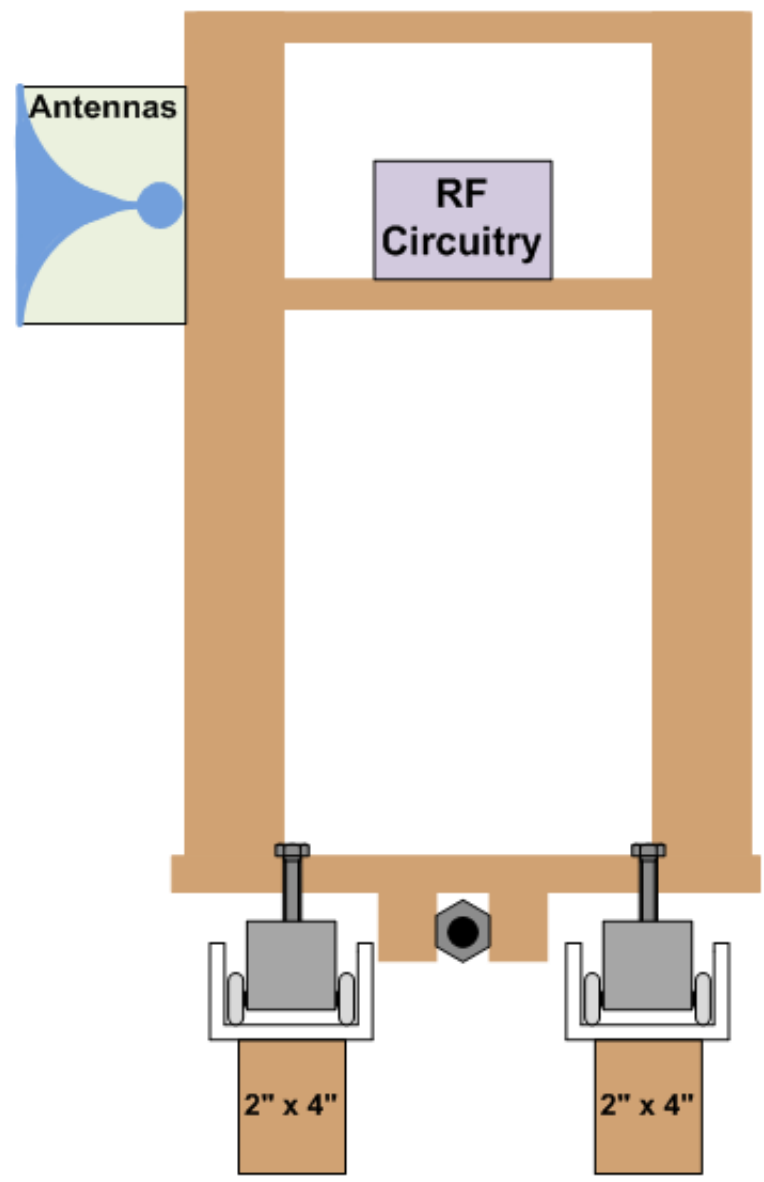

SWE VIEW

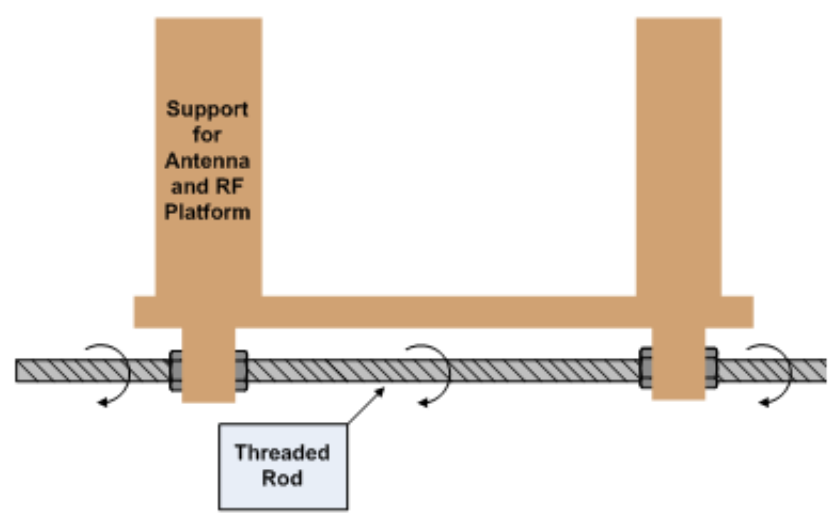

FIGURE 4-3: MOVEMENT SYSTEM DESIGN \#2 
A "closet door" trolley is installed on each platform corner. For guide rails, both PVC pipes were replaced with two $10 \mathrm{ft}$ length, $1 / 2$ " right angle aluminum tracks to provide a channel for the trolley wheels.

This design did not work due to slow speed and unreliable motion. The wheels developed friction against the guide rails which impeded the rolling motion. The screw nut separates from its wood mounting location on the platform and does not facilitate disassembly and radar platform relocation.

FRONT VIEW

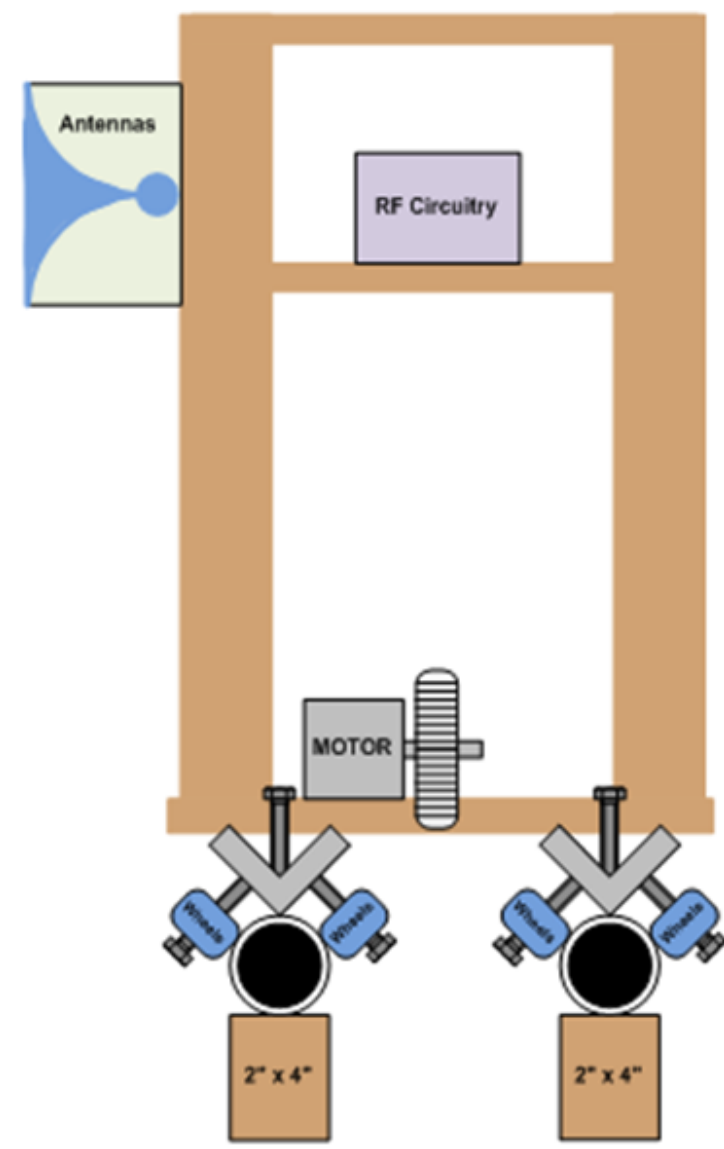

\section{SIDE VIEW}

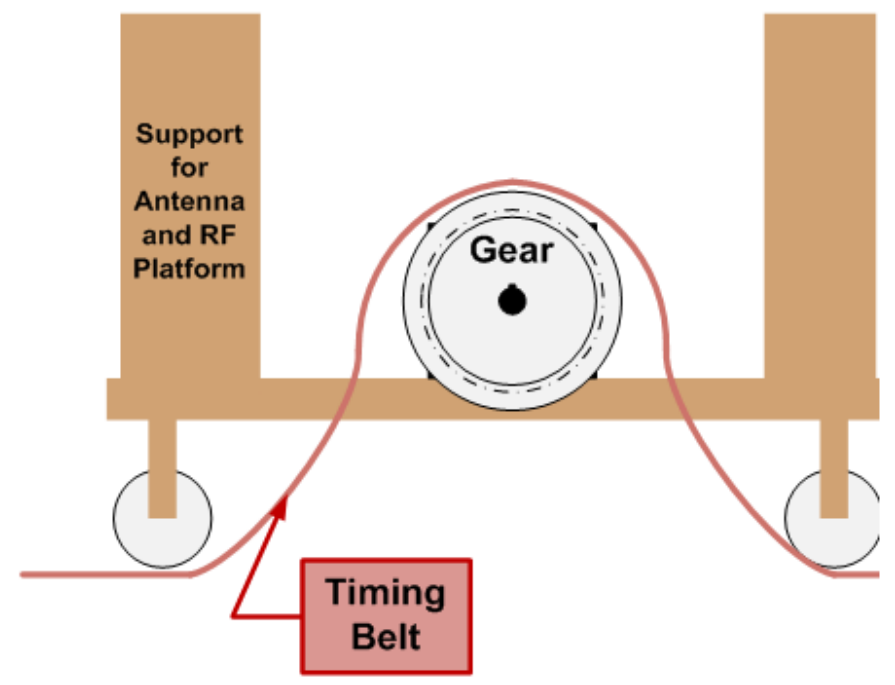

FIGURE 4-4: MOVEMENT SYSTEM FINAL DESIGN 
In this design, the motor is mounted on the moving platform and includes a gear connected to a 10 foot timing belt running along the guide rails. The PVC pipes are mounted on top of the guide rails. Instead of sliding on top of the pipes, skateboard wheels were mounted to both sides of right angle aluminum sections to roll on the sides of the PVC pipes, see Figure 4-4. This third design worked the best of all and was selected as the final configuration. The final design provides speeds of 3 meters per minute while limiting overall system weight to 75 pounds.

One major deviation from airplane or satellite motion is the proposed system's discrete step platform motion. Between each positioner movement, a pulse is transmitted and stored in memory before continuing to the next point along the rail. This may seem to be a substantial difference; however, even in the case of airplanes and satellites, it is a valid approximation to assume vehicle movement in this manner during data collection [6].

\subsection{MOTOR AND CONTROL CIRCUITRY}

Motor control circuitry achieves precise movements to guarantee specific locations for each data point along the rail. Movement is controlled by a stepper motor with 200 steps per revolution and 125 ounce-inches of torque. A stepper motor is a form of brushless DC electric motor that rotates to a specific angle in response to the rising edge of an applied voltage pulse sequence. This characteristic is important for location repeatability for each data point along the rail. Based on the desired number of data points, LabView calculates the required number of motor control pulses for each discrete movement and sends required commands to the microcontroller. However, the required motor current (1.6A) substantially exceeds the micro-controller's supply current capability: 50mA. To 
overcome this problem, a motor driving circuit (maximum current $2 \mathrm{~A}$ ) is used. This unit translates motor control signals to pulse width modulation (PWM) for accurate stepper motor movements.

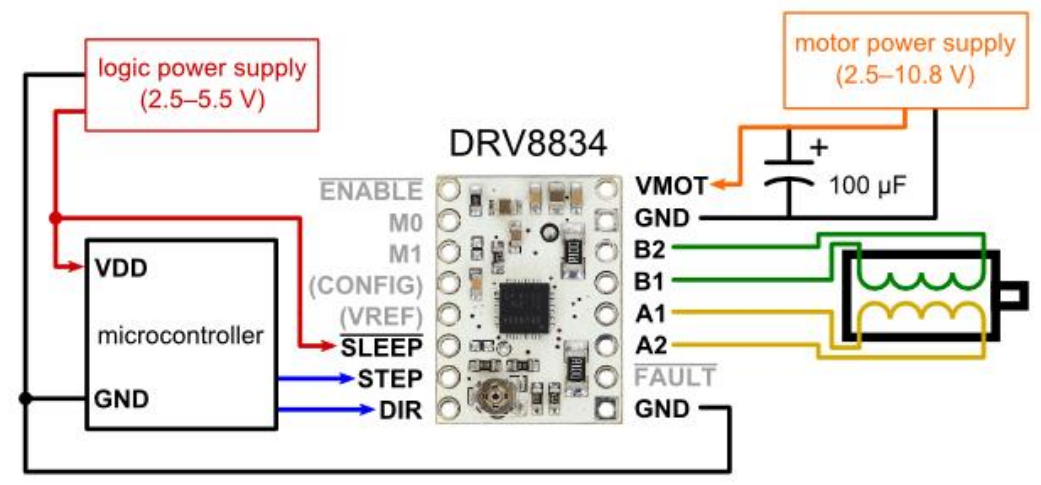

FIGURE 4-5: TI DRV8834 BREAKOUT BOARD WIRING DIAGRAM, STEPPER MOTOR CONTROL

The Texas Instruments DRV8834 stepper motor driver was designed to drive low voltage $(3 \mathrm{~V}, 2 \mathrm{~A})$ stepper motors. Figure 4-5 illustrates the required connections using the TI DRV8834 motor control IC mounted to a breakout board for system integration. The micro-controller toggles the IC digital logic pins while the IC is also connected to a high current capacity (2.5A) power supply and the stepper motor. For each required stepper motor rotation, the step pin is toggled by the micro-controller from low to high. Repeated toggling, a square wave, results in continuous motion. 


\subsection{ANTENNA TOPOLOGY CONSIDERATION}

As shown in section 1.3.1 above, a chirp signal bandwidth of $1 \mathrm{GHz}$ is required to achieve an image range resolution on the order of $15 \mathrm{~cm}$. However, the VCO used in the RF frontend is capable of sweeping greater than $1 \mathrm{GHz}$ bandwidth, allowing even finer image range resolution. The antennas must operate over the entire VCO frequency range, over $2 \mathrm{GHz}$ bandwidth. Few antenna topologies offer the bandwidth and directivity required for wide band radar applications.

Table 5-1 summarizes design criteria to initially select radar system antennas. Design parameters are prioritized below based on system operation importance.

TABLE 5-1: ANTENNA DESIGN REQUIREMENTS; FROM GREATEST (1) TO LEAST (5) IMPORTANCE

\begin{tabular}{|l|l|}
\hline Design Parameters & Parameter Importance \\
\hline $\begin{array}{l}\text { Center Frequency 2GHz, } \\
\text { Wide Bandwidth (1-3GHz) }\end{array}$ & 1 \\
\hline Single Main Lobe Radiation & 1 \\
\hline Gain (dBi) & 2 \\
\hline Half-Power Beamwidth (degrees) & 2 \\
\hline Lightweight & 3 \\
\hline Small Form Factor & 3 \\
\hline Low-Cost & 4 \\
\hline
\end{tabular}

Table 5-1 specifies an antenna topology that requires a 100\% bandwidth (ratio of bandwidth to center frequency) and a single radiation pattern main lobe at low cost. After 
reviewing multiple wideband radar systems [1], the Vivaldi flare antenna was chosen as the best topology for this application, due to wideband response, light weight, and small form factor.

\subsection{DESIGN GEOMETRY}

The Vivaldi flare is a linearly tapered slot antenna (LTSA). Due to their flared geometry, see Figure 5-1, these antennas maintain efficient radiation and constant beam-width over a wide frequency range.

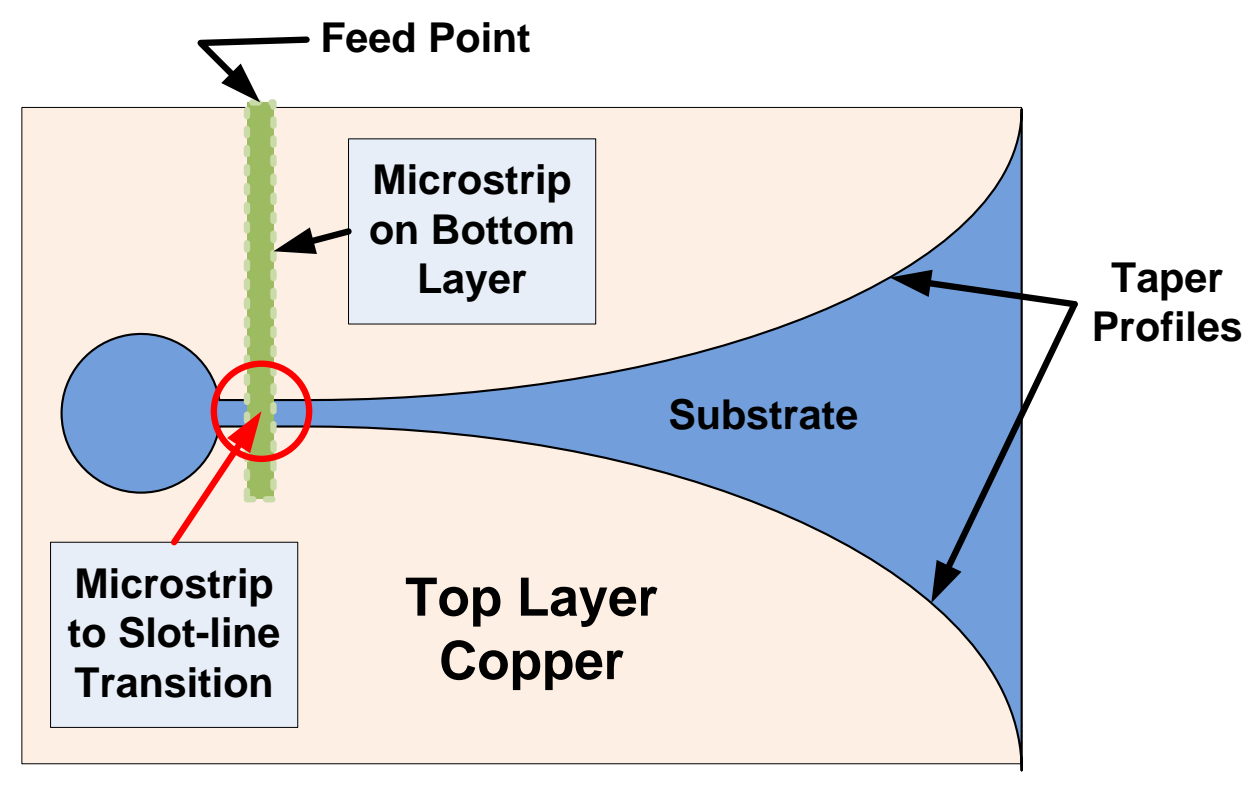

FIGURE 5-1: VIVALDI FLARE GEOMETRY

The Vivaldi flare and similar geometries are leaky wave antennas due to their radiation mechanism. When the transmit signal couples from the micro-strip to the slot-line gap, energy is contained between the two conductor taper profiles. As the wave travels along the antenna, the taper profiles grow steadily apart. At the point where the tapers are approximately one free space wavelength apart, the energy begins to radiate. These 
antennas therefore radiate over extremely wide bandwidths; the antenna's wide end (mouth opening in Fig. 5-2) is $\lambda / 2$ in free space at the lowest operating frequency [2]. The slot line transition width (throat width) must be one free space wavelength for the highest operating frequency. Figure 5-2 shows the antenna's flare portion growing wider across the antenna's length. The red section in Figure 5-2 corresponds to the feed line located on the antenna's bottom layer. The blue area is located on the antenna's top layer. This taper follows the exponential function shown in equations 5-1, 5-2, and 5-3 below [2].

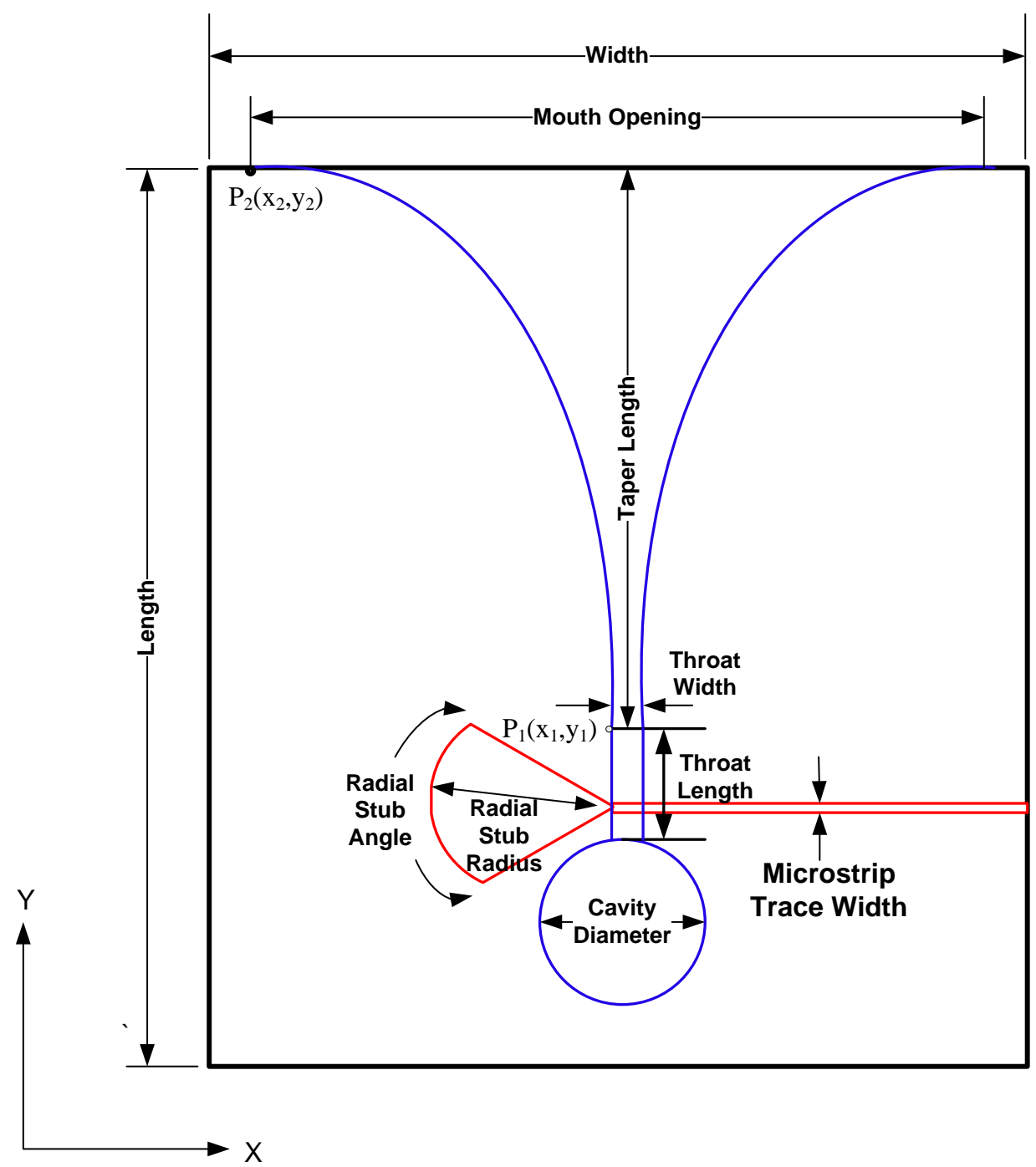

FIGURE 5-2: VIVALDI FLARE, ANNOTATED GEOMETRY 


$$
\begin{gathered}
f(x)=c_{1} e^{R x}+c_{2} \\
c_{1=} \frac{p_{2 y}-p_{1 y}}{e^{R * p_{2 x}}-e^{R * p_{1 x}}} \\
c_{2}=\frac{\left(p_{1 y} * e^{R * p_{2 x}}\right)-\left(p_{2 y} * e^{R * p_{1 x}}\right)}{e^{R * p_{2 x}}-e^{R * p_{1 x}}} \\
R=\text { taper rate } \\
P_{1}(x, y)=\text { Starting } x, y \text { coordinate of flare/taper } \\
P_{2}(x, y)=\text { Ending } x, y \text { coordinate offlare/taper }
\end{gathered}
$$

The feed mechanism has no direct physical connection to the antenna's radiating area, which contains the copper sheet cut-out flare defined in equations 5-1 through 5-3. The opposite side contains a microstrip trace that terminates in an open circuit radial stub (broadband). The microstrip signal energy couples through the substrate to excite the radiating flare.

\subsection{DESIGN PROCEDURE}

Since the SAR system operates on the ground, there was flexibility on physical antenna size. The main constraint is the fabrication method. To maintain project costs below $\$ 6000$, the antennas are fabricated using university-available methods. An LPKF milling machine was used for antenna milling. It is limited to antennas less than 9" by 11.5 ".

Antenna gain and beam-width are important constraints for maximizing SAR range. Increasing the antenna gain $(\mathrm{G})$ decreases antenna beam-width (BW in radians) as defined by [10]: 


$$
G=\frac{4 \pi}{B W_{\theta e l} B W_{\phi a z}}
$$

Azimuthal direction antenna beam-width is inversely proportional to strip-map SAR maximum range. The tradeoff between gain and azimuthal beam-width balances maximum target detection range against maximum azimuthal image area.

The final Vivaldi antenna constraint is return loss, $-20 * \log _{10}\left(\left|S_{11}\right|\right)$. Acceptable performance was set to $-10 \mathrm{~dB}$ return loss over the $1 \mathrm{GHz}$ to $3 \mathrm{GHz}$ bandwidth. This specification ensures efficient (>90\%) power transfer efficiency between RF circuitry and the antennas.

\subsection{CANDIDATE DESIGN CONFIGURATIONS}

Vivaldi antenna development [3] includes optimum physical size. Optimum Vivaldi antenna operation is achieved with length greater than $1 \lambda$ and an aperture width (mouth opening in Figure 5-2) greater than $\lambda / 2$ at the minimum frequency. With a frequency range from 1 to $3 \mathrm{GHz}$, the required length is greater than 11.8 inches with an aperture width greater than 5.9 inches. This exceeds milling machine capabilities. The Vivaldi antenna's physical size is limited to 8.5 " by 11 " to accommodate the milling machine; the theoretical geometry must be adjusted using simulations in CST Microwave Studio.

\subsection{COMPARISON ANALYSIS/SIMULATIONS}

Geometric parameters with greatest antenna operation effects are length, width, and throat width, see Figure 5-2. To start simulations, the initial length is set to one free space wavelength at the lowest operating frequency $\left(\mathrm{f}_{\min }=2 \mathrm{GHz}\right), \lambda_{\min }=\mathrm{c} / \mathrm{f}_{\min }=0.15 \mathrm{~m}=5.9 \mathrm{in}$. The initial antenna width is $\frac{\lambda}{2}$. The throat width is set to the incoming $50 \Omega$ micro-strip 
feed width at the antenna center frequency $2 \mathrm{GHz}$. Using Agilent ADS Line-calc, the initial throat width is 70.36 mil. Optimization was performed to minimize $\left|\mathrm{S}_{11}\right|<-15 \mathrm{~dB}$ from 1 to $3 \mathrm{GHz}$. Using optimization results for length, width, and throat width, a second optimization was performed for the remaining antenna parameters (mouth opening, taper length, throat length, cavity diameter, radial stub radius, radial stub angle), all with the same goal. From the first optimization attempt, throat width and microstrip feedline width prevented the $2 \mathrm{GHz}$ bandwidth. To remedy this, alternate broadband microstrip transmission line topologies were investigated including broadband tapered microstrip lines [5]. A tapered line's characteristic impedance changes along the line's length. This addition was incorporated into the design by using a $50 \Omega$ to $100 \Omega$ (69.8 and $24.1 \mathrm{mil}$ width) tapered line. Three antenna simulations were performed, using the tapered microstrip feedline.

All designs were simulated using CST microwave studio; the final design was based on $\left|S_{11}\right|$ performance and manufacturability, see Table 5-2. The first design exceeds milling machine capabilities, but provides the best performance. The second design meets milling machine requirements but not return loss. The final design meets milling machine constraints and exhibits return loss greater than 9dB across 1 to $3 \mathrm{GHz}$. Figure 5-3 shows simulated return loss for the three antenna designs.

Note: $\mathrm{RL}=-20 \log (|\Gamma|) \mathrm{dB}$ 
TABLE 5-2:VIVALDI FLARE PERFORMANCE COMPARISON

\begin{tabular}{|c|c|c|c|c|c|c|}
\hline $\begin{array}{c}\text { Antenna } \\
\text { Designs }\end{array}$ & $\begin{array}{c}\text { Minimum } \\
\text { Return Loss }\end{array}$ & $\begin{array}{c}\text { Gain @ } \\
2 \mathrm{GHz}\end{array}$ & $\begin{array}{c}\text { Physical } \\
\text { Length }\end{array}$ & $\begin{array}{c}\text { Physical } \\
\text { Width }\end{array}$ & $\begin{array}{c}\text { Electrical } \\
\text { Length }\end{array}$ & $\begin{array}{c}\text { Electrical } \\
\text { Width }\end{array}$ \\
\hline Design \#1 & $-12.0 \mathrm{~dB}$ & $10.4 \mathrm{dBi}$ & $14 ”$ & $13 ”$ & $1.18 \lambda$ & $1.10 \lambda$ \\
\hline Design \#2 & $-8.3 \mathrm{~dB}$ & $8.6 \mathrm{dBi}$ & $11 ”$ & $9 ”$ & $0.93 \lambda$ & $0.76 \lambda$ \\
\hline Design \#3 & $-8.9 \mathrm{~dB}$ & $7.9 \mathrm{dBi}$ & $10 ”$ & $8 ”$ & $0.84 \lambda$ & $0.68 \lambda$ \\
\hline
\end{tabular}

Vivaldi Flare Return Loss Comparison

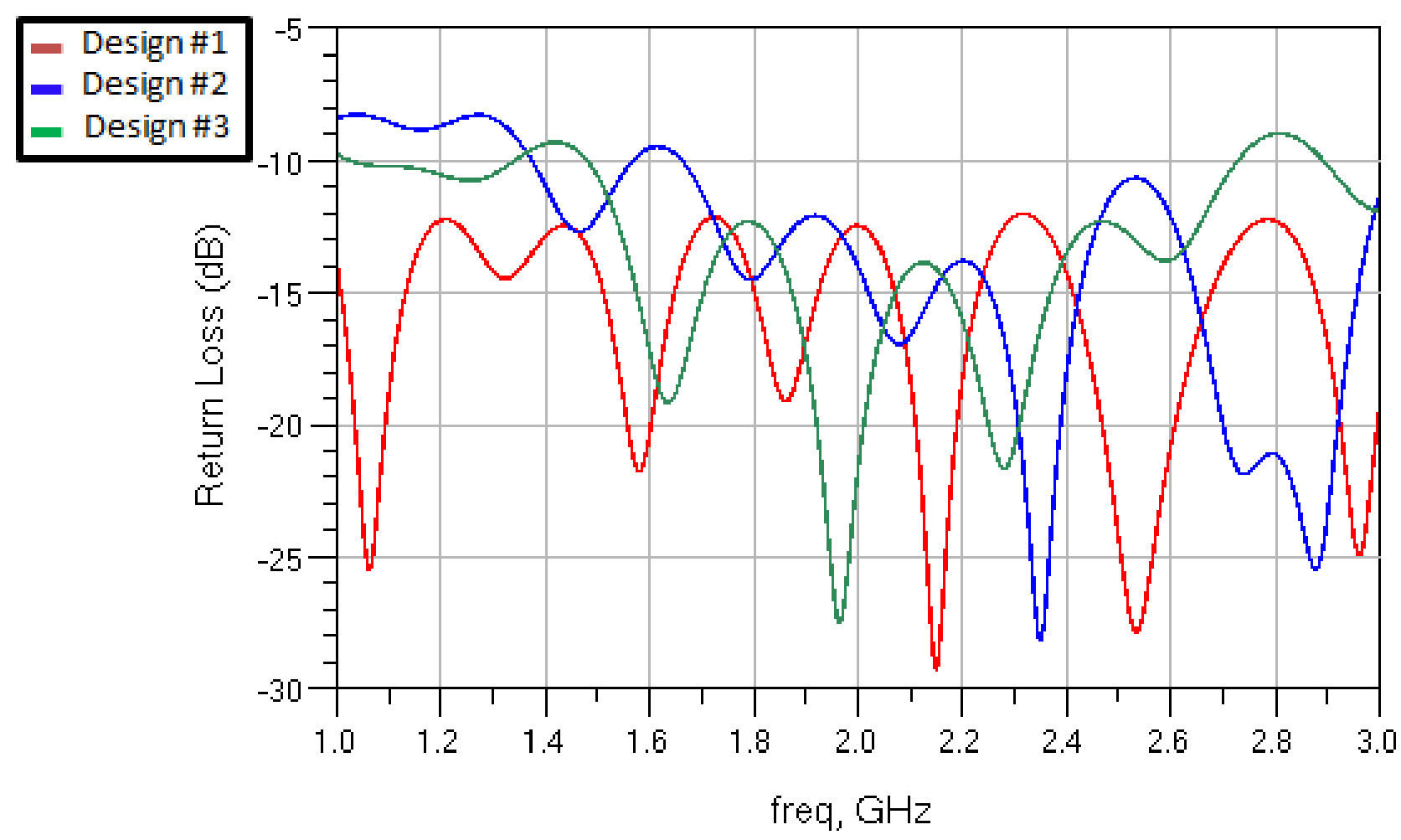

FIGURE 5-3: VIVALDI FLARE SIMULATED RETURN LOSS COMPARISON, THREE CANDIDATE DESIGNS

Although Design \#1 has the best performance, its size exceeds milling machine limits.

Designs \#2 and \#3 both meet the size criteria; however, Design \#2 has greater gain than 
Design \#3 and less optimal return loss. Because gain is inversely proportional to half power beam width (section 5-1) Design \#3 was selected for fabrication. Table 5-3 shows the final Vivaldi flare antenna dimensions.

TABLE 5-3: FINAL VIVALDI FLARE DESIGN GEOMETRY DIMENSIONS

\begin{tabular}{|c|c|}
\hline Length & $8 ”$ \\
\hline Width & $10 ”$ \\
\hline Mouth Opening & $8.935 "$ \\
\hline Taper Rate (R) & 0.235 \\
\hline Taper Length & $6.176 "$ \\
\hline Throat Width & 95.9 mil \\
\hline Throat Length & 948.7 mil \\
\hline Cavity Diameter & $763.1 \mathrm{mil}$ \\
\hline Backwall Offset & 112.5 mil \\
\hline Radial Stub Angle From Vertical & 0.15 radians \\
\hline Radial Stub Angle & 1.95 radians \\
\hline Radial Stub Radius & $507.7 \mathrm{mil}$ \\
\hline Microstrip Edge Trace Width & 69.9 mil \\
\hline $\begin{array}{c}\text { Microstrip Radial Stub Termination } \\
\text { Trace Width }\end{array}$ & $24.1 \mathrm{mil}$ \\
\hline
\end{tabular}

Figure 5-4 below shows the final design's simulated radiation pattern. 


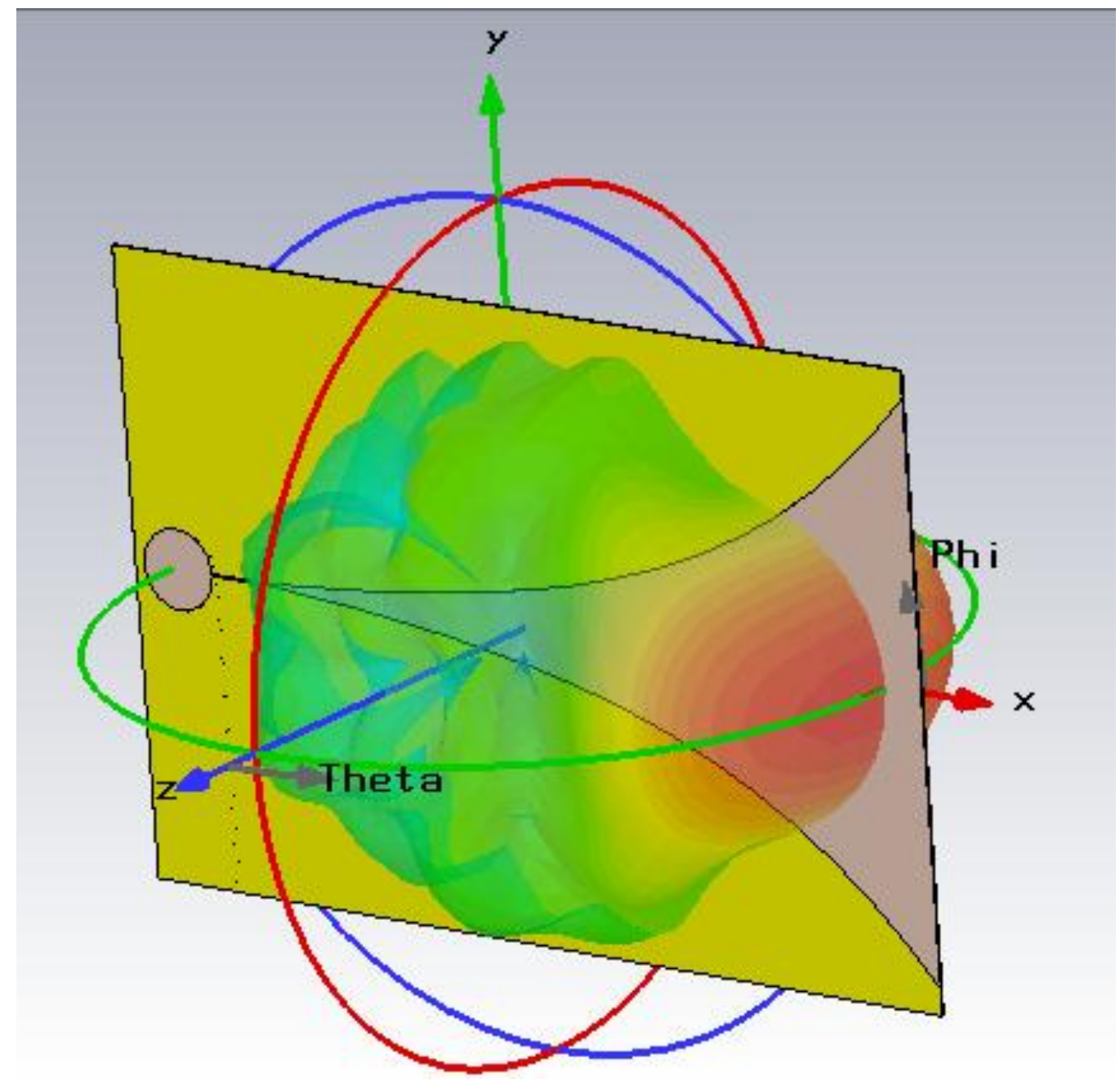

FIGURE 5-4: SIMULATED VIVALDI FLARE ANTENNA GEOMETRY AND RADIATION PATTERN

\subsection{FABRICATION PROCESS}

Transmit and receive antennas were fabricated with Table 5-3 dimensions. An LPKF

ProtoMat S62 milling machine was used to mill the copper shapes from double sided 1/2 ounce copper clad Rogers Duroid 4350b. After CST microwave studio refinements, three .GBR (Gerber) files were imported into LPKF milling machine software; top layer, 
shape outline and bottom layer. Total fabrication time was approximately 30 minutes per antenna.

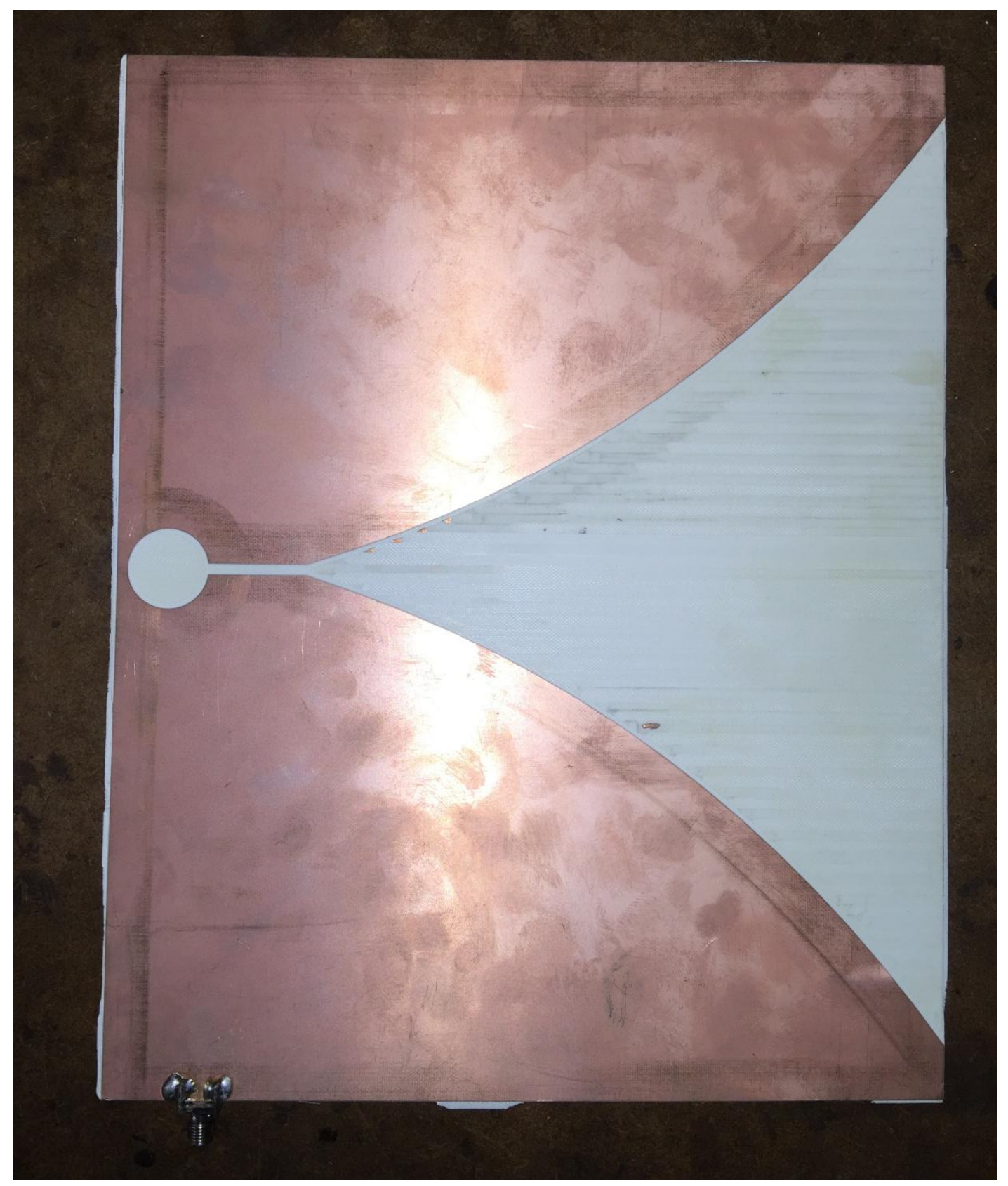

FIGURE 5-5: COMPLETED VIVALDI FLARE ANTENNA, FRONT SIDE 


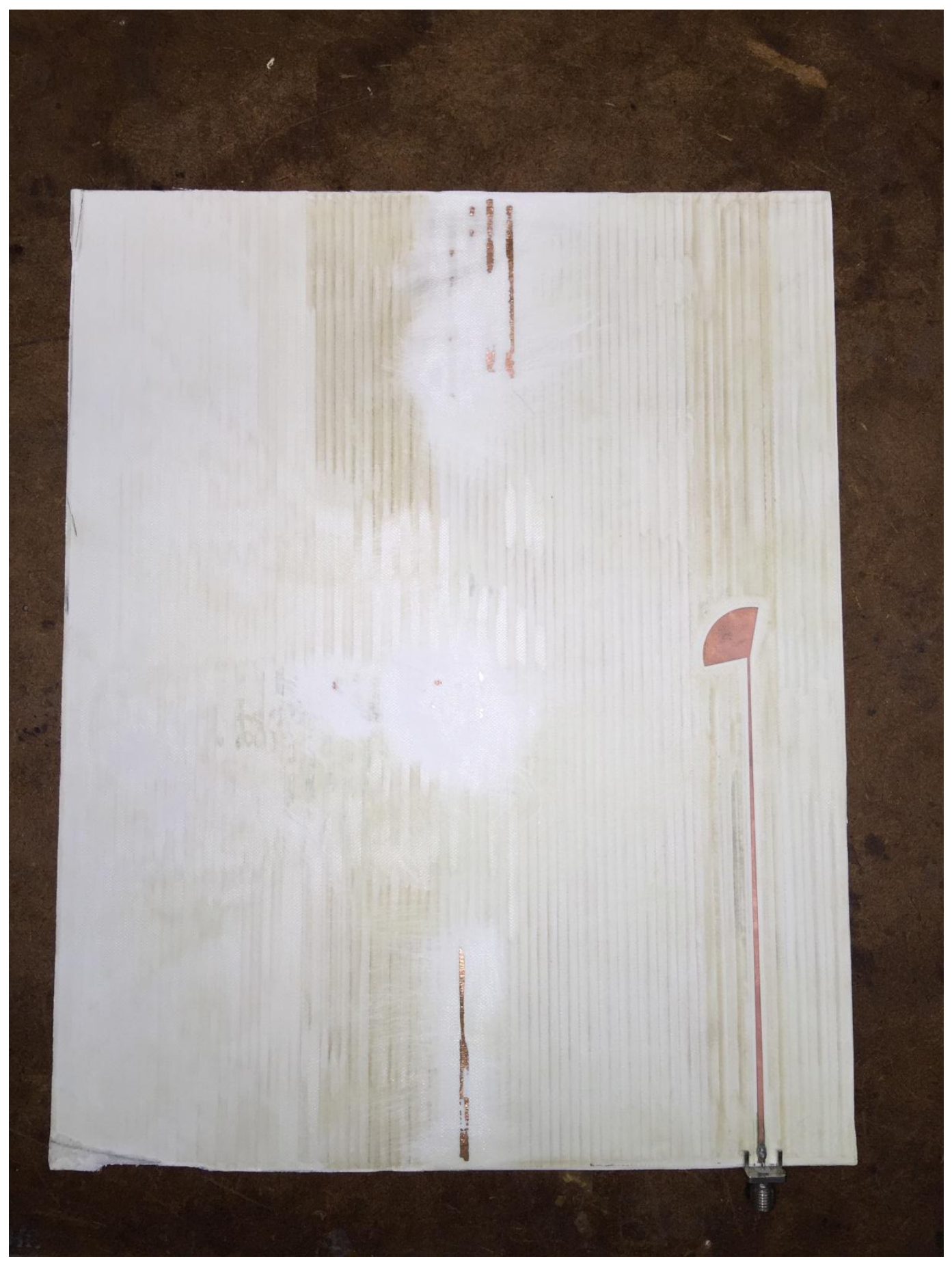

FIGURE 5-6: COMPLETED VIVALDI FLARE ANTENNA, REVERSE SIDE

Figures 5-5 and 5-6 above show the front and back side of the finished antenna. On the back side, the milling machine failed to remove all copper cladding from "mill out" areas. 
Section 5.7 below discusses possible effects of the stray copper pieces on antenna performance.

\subsection{RESULTS}

Return loss, gain, and radiation pattern measurements were recorded using an anechoic chamber and standard gain horns. Figures 5-7 and 5-8 show the measured return loss and peak gain compared to simulated results.

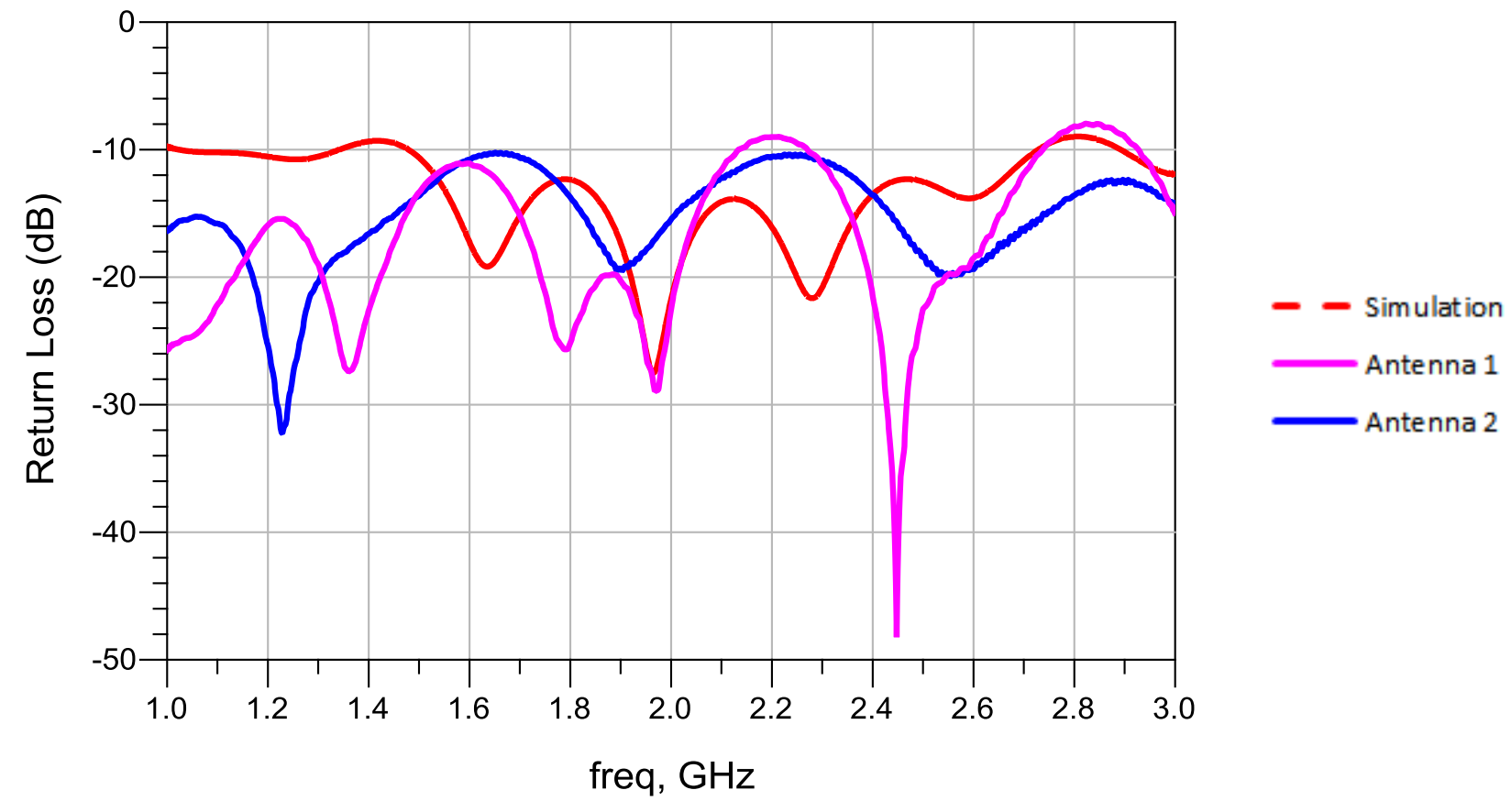

FIGURE 5-7: SIMULATED VS. MEASURED RETURN LOSS COMPARISON FOR FINAL FABRICATED VIVALDI FLARE ANTENNA PAIR

From the simulation, antenna return loss was less than $-10 \mathrm{~dB}$ across the 1 to $3 \mathrm{GHz}$ bandwidth. The fabricated antennas show similar performance, with the measured return loss of Antenna 2 less than $-10 \mathrm{~dB}$ across the entire $2 \mathrm{GHz}$ bandwidth. The return loss of Antenna 1 is less than $-10 \mathrm{~dB}$ across the $2 \mathrm{GHz}$ bandwidth except for $2.2 \mathrm{GHz}$ and 2.8 GHz. Major return loss performance differences between the two antennas can be 
attributed to manufacturing variability. Because the feed geometry is a relatively small copper structure, the LPKF milling machine did not consistently remove all copper on the antenna's feed side. Attempting to remove the copper manually resulted in substrate removal, which altered the antenna's performance. The copper remnants may couple to the antenna's main flare section causing $\left|\mathrm{S}_{11}\right|$ to degrade. Also, subtle changes in milling bit "sharpness" during the copper removal process may have resulted in dielectric substrate thickness variation across the antenna. With variable substrate thickness under the feed and radiating areas, the micro-strip transmission line's characteristic impedance detunes from original design values, also resulting in $\left|S_{11}\right|$ degradation.

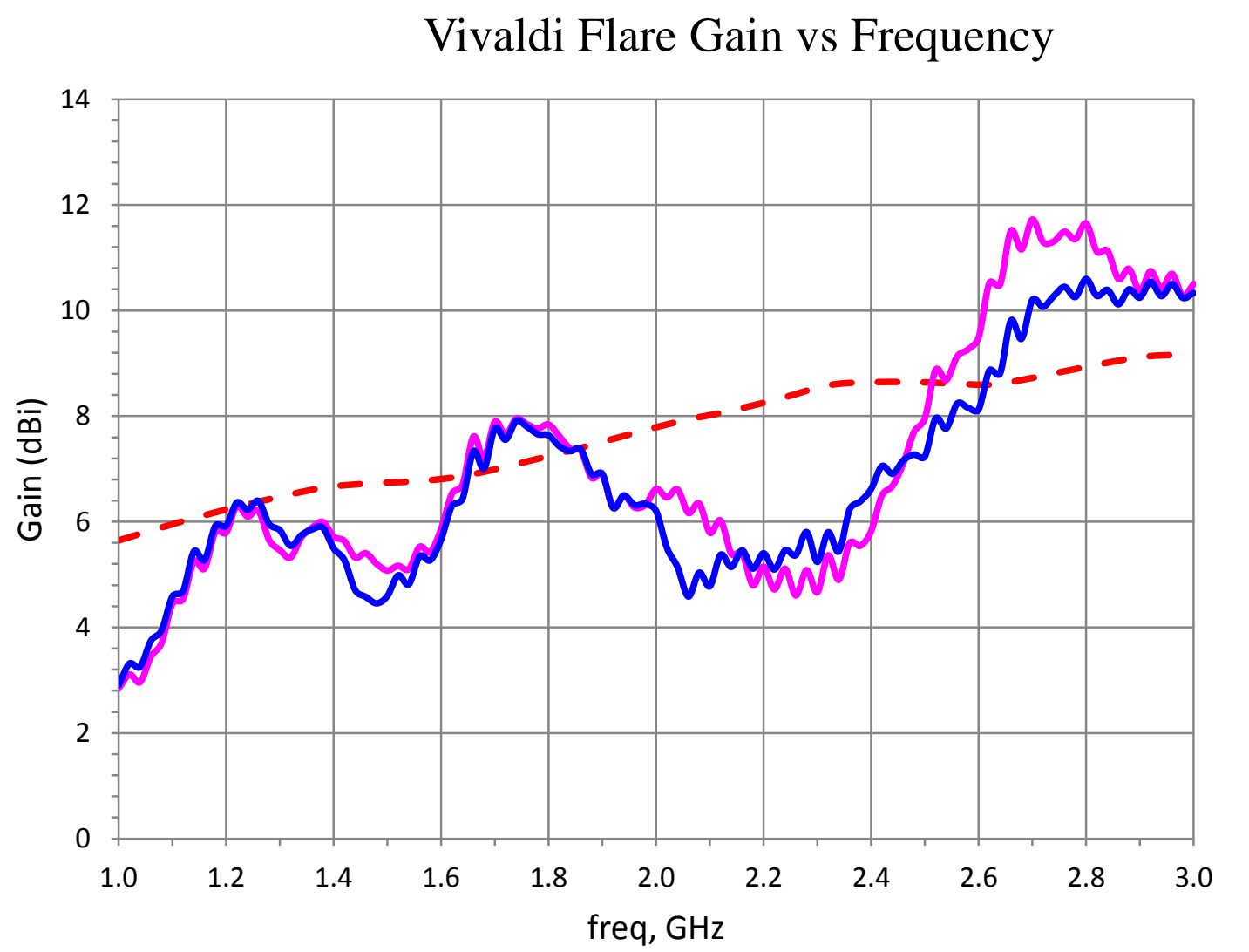

- Simulation

Antenna 1

Antenna 2

FIGURE 5-8: SIMULATED VS. MEASURED GAIN COMPARISON FOR TWO FABRICATED VIVALDI FLARE ANTENNAS 
Fabricated antenna gain measurements were recorded in an anechoic chamber on the Cal Poly campus. Simulated gain data monotonically increases from approximately $6 \mathrm{dBi}$ to $9 \mathrm{dBi}$ across the 1 to $3 \mathrm{GHz}$ band. While measured results shows $2 \mathrm{~dB}$ fluctuations in peak gain across the band (consistent between the antenna pair), the overall gain trend follows simulated expectations with a maximum difference of $3.5 \mathrm{dBi}$ at $2.1 \mathrm{GHz}$.

The anechoic chamber was also used to measure fabricated antenna radiation patterns at $2 \mathrm{GHz}$. Figure 5-9 shows the antenna geometry and coordinate system for the measured radiation pattern.

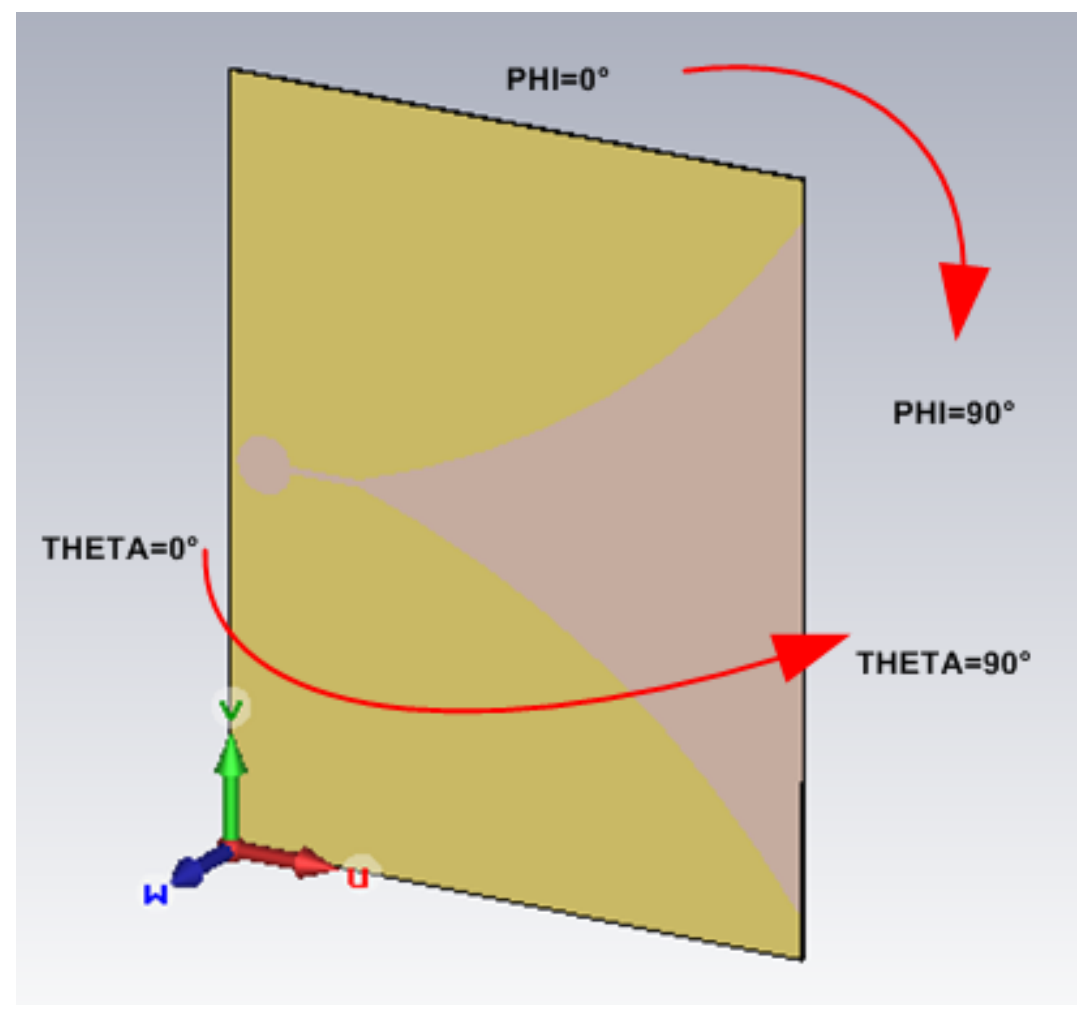

FIGURE 5-9: VIVALDI FLARE RADIATION PATTERN COORDINATE SYSTEM: EPLANE (PHI SCAN) AND H-PLANE (THETA SCAN) 


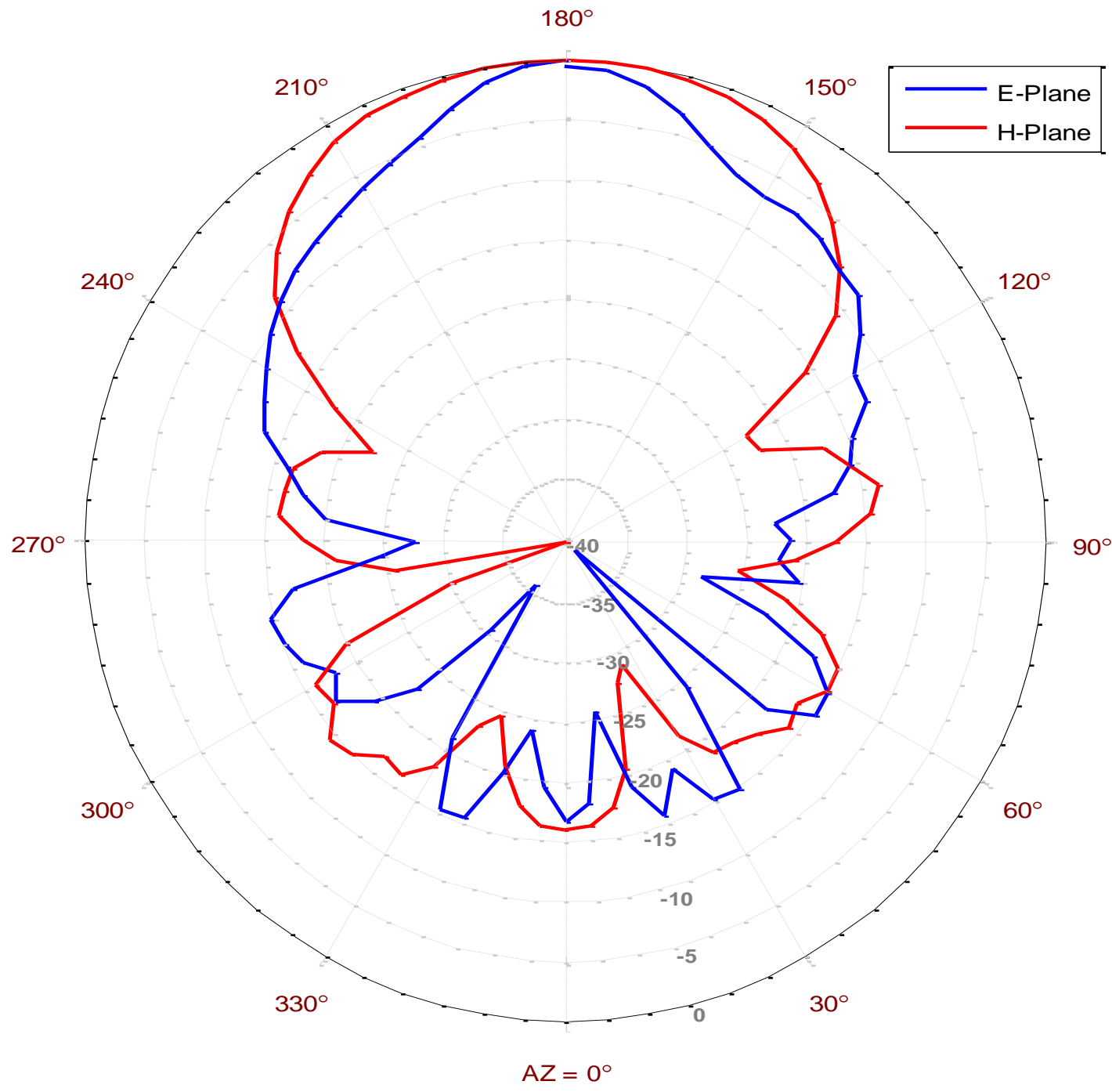

FIGURE 5-10: MEASURED VIVALDI FLARE \#1 RADIATION PATTERN: CO POL H PLANE (RED), CO POL E PLANE (BLUE)

The measured Vivaldi flare radiation pattern in Figure 5-10, shows correlation with the simulated pattern in Figure 5-4. In both simulated and measured patterns, the main lobe is squinted in the E-plane; broad in the H-plane. The half power beam width in the H-plane is $62^{\circ}$ and $30^{\circ}$ in the E-plane. The half power beam width in the H-plane is also the azimuthal domain beam width during data collection. 
The RF sub-system transmits and receives chirp pulses, see section 3.3.1. RF section design refinements reduce development and troubleshooting time. SMA connected components (modular method) allows component interchanges among signal chain locations.

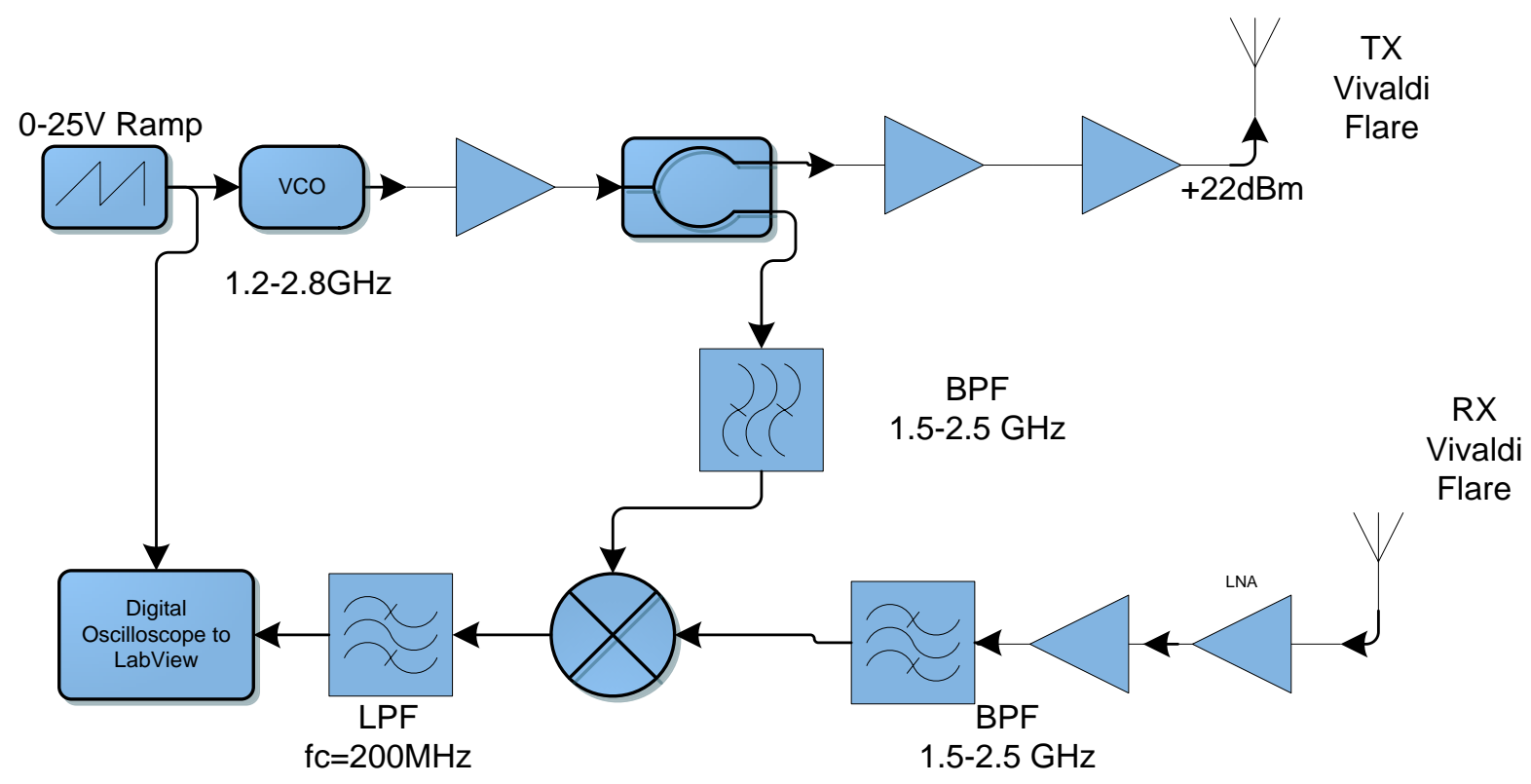

FIGURE 6-1: RF SIGNAL CHAIN BLOCK DIAGRAM

The voltage ramp generating circuit is connected to the Mini-Circuits ZX95-2800-S+ VCO to produce a chirp signal with frequency range 1.20 to $2.95 \mathrm{GHz}$. The VCO drives a micro-strip power splitter connected to a band pass filter and two Mini-Circuits ZX60V63+ amplifiers to create sufficient transmit power $(22 \mathrm{dBm})$. 


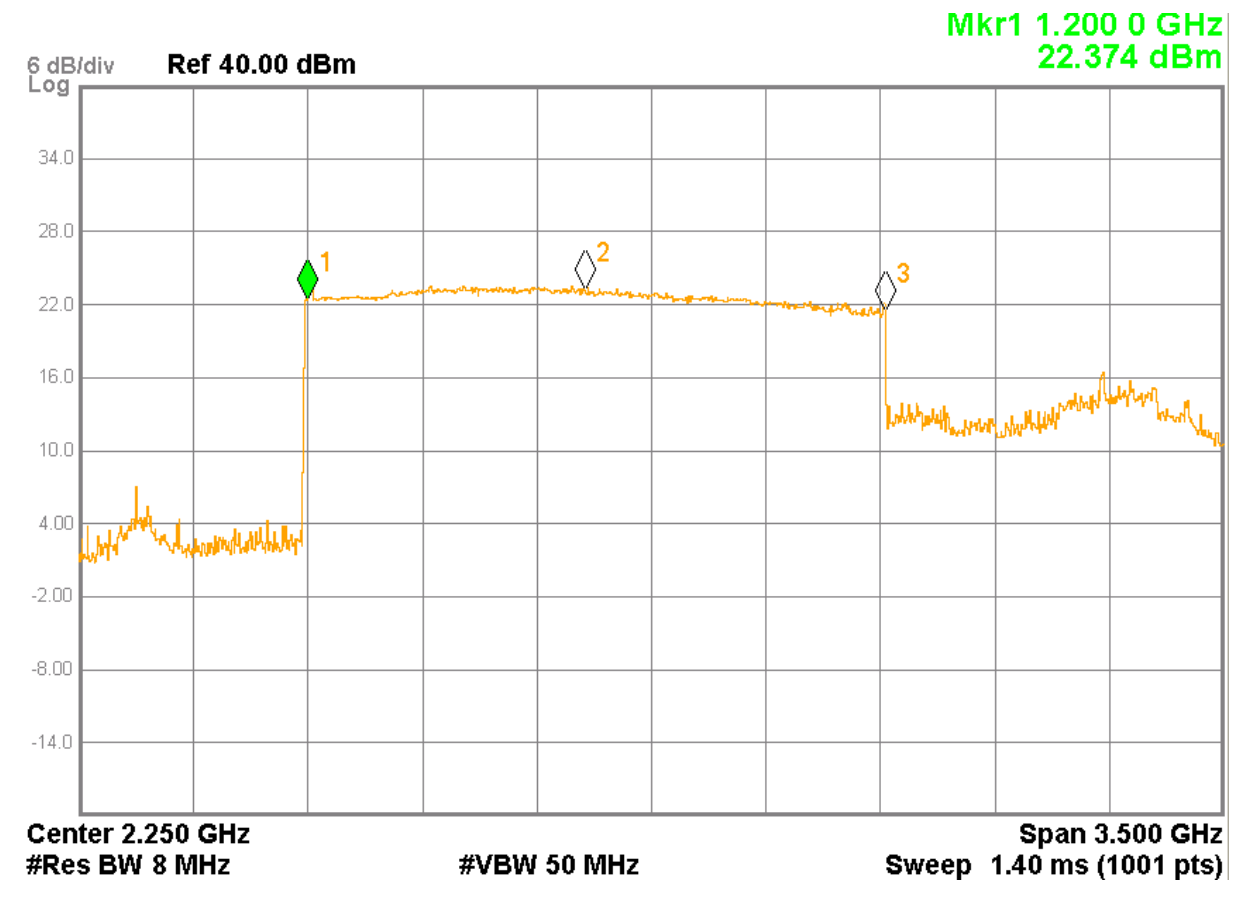

FIGURE 6-2: MEASURED TRANSMITTED SIGNAL FREQUENCY SPECTRUM

The transmitted signal spectrum is shown in Figure 6-2. Transmit power varies by $1.5 \mathrm{~dB}$ over the $1.75 \mathrm{GHz}$ bandwidth. In order to achieve a flat transmit spectrum, the output stage amplifiers are driven into compression; output amplifier power varies approximately $4 \mathrm{~dB}$ over its $1 \mathrm{~dB}$ output compression point of $18 \mathrm{dBm}$. Driving the final amplifier stage into compression produces large intermodulation products. However, the receive signal chain band pass filter and the pulse compression operation outlined in section 6.1 (next section), attenuate these intermodulation products to minimize system performance impact.

In the receive signal chain, the receive antenna is connected directly to the Mini-Circuits PSA4-5043+ LNA (low noise amplifier) followed by another Mini-Circuits ZX60-V63+ amplifier and wideband band pass filter. The received signal frequency (1.20 to 
$2.95 \mathrm{GHz}$ ) is beyond sampling capabilities for effective data collection. Even if the signal is down converted to baseband frequencies, the signal covers the DC-1.75 GHz bandwidth. To overcome this wide bandwidth signal sampling problem, "pulse compression" is implemented by connecting transmit and receive signals to the mixer LO and RF ports, respectively.

\subsection{PULSE COMPRESSION}

Pulse compression is used in many wideband radar systems to reduce data collection hardware requirements. As discussed in Range Doppler Algorithm (section 1.3), range (or pulse) compression is the first algorithm step. In this case, range compression is accomplished in hardware using a mixer instead of software processing. The theory behind hardware range compression is described below.

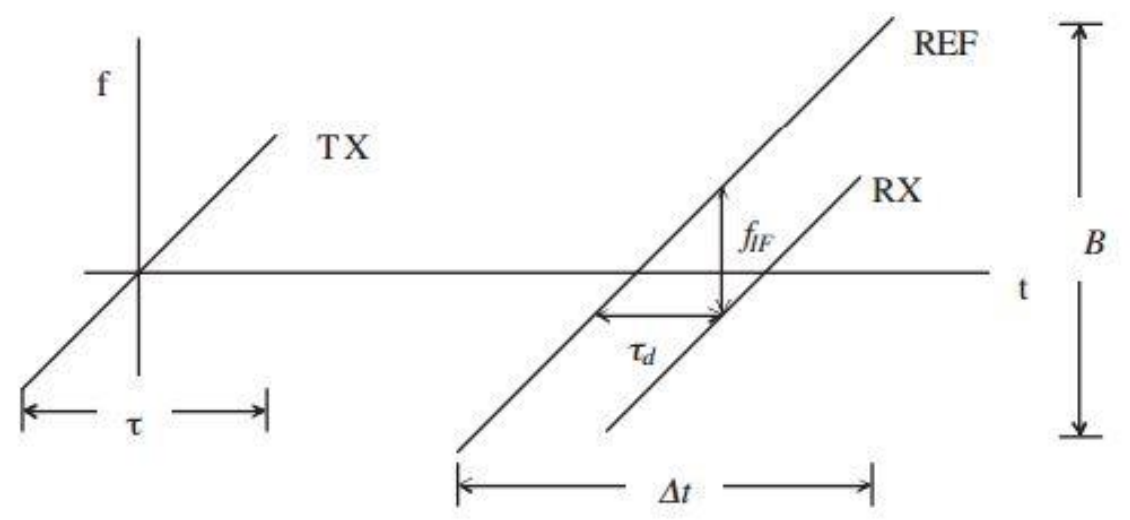

FIGURE 6-3: PULSE COMPRESSION TIMING DIAGRAM

The left side of Figure 6-3 defines the transmitted waveform (TX) with pulse duration $\tau$ over time (t) vs. frequency (f). This pulse is reflected from an object and received. The received waveform $(\mathrm{RX})$, denoted $\mathrm{REF}$ for reference, is received $\tau_{\mathrm{d}}$ seconds after the beginning of the TX pulse is transmitted. Assuming that the reference TX pulse is still 
being transmitted when the received pulse returns, the instantaneous received pulse frequency will differ from the current TX pulse. This frequency difference is defined by:

$$
f_{I F}=\frac{B}{\Delta t} \tau_{d}
$$

where B is the total TX pulse bandwidth and $\Delta \mathrm{t}$ is the TX chirp pulse duration. Since the distance to the furthest target for this radar system is less than 100 feet, the total time delay, $\tau_{\mathrm{d}}$, is less than $200 \mathrm{~ns}$.

$$
\tau_{d}=\frac{2 * \text { distance }}{c}
$$

If the transmit pulse duration is $5 \mu \mathrm{s}$, with a $1.75 \mathrm{GHz}$ bandwidth, the resulting difference frequency $\mathrm{f}_{\mathrm{IF}}$ is a maximum of $70 \mathrm{MHz}$. From this example case, the range compression signal's maximum possible frequency is indirectly determined by the pulse sweep rate. 
Because range compression is implemented in hardware (section 6.1), a modified version of the Range Doppler Algorithm (section 1.3) was attempted. A block diagram of the successfully implemented signal processing procedure is shown below.

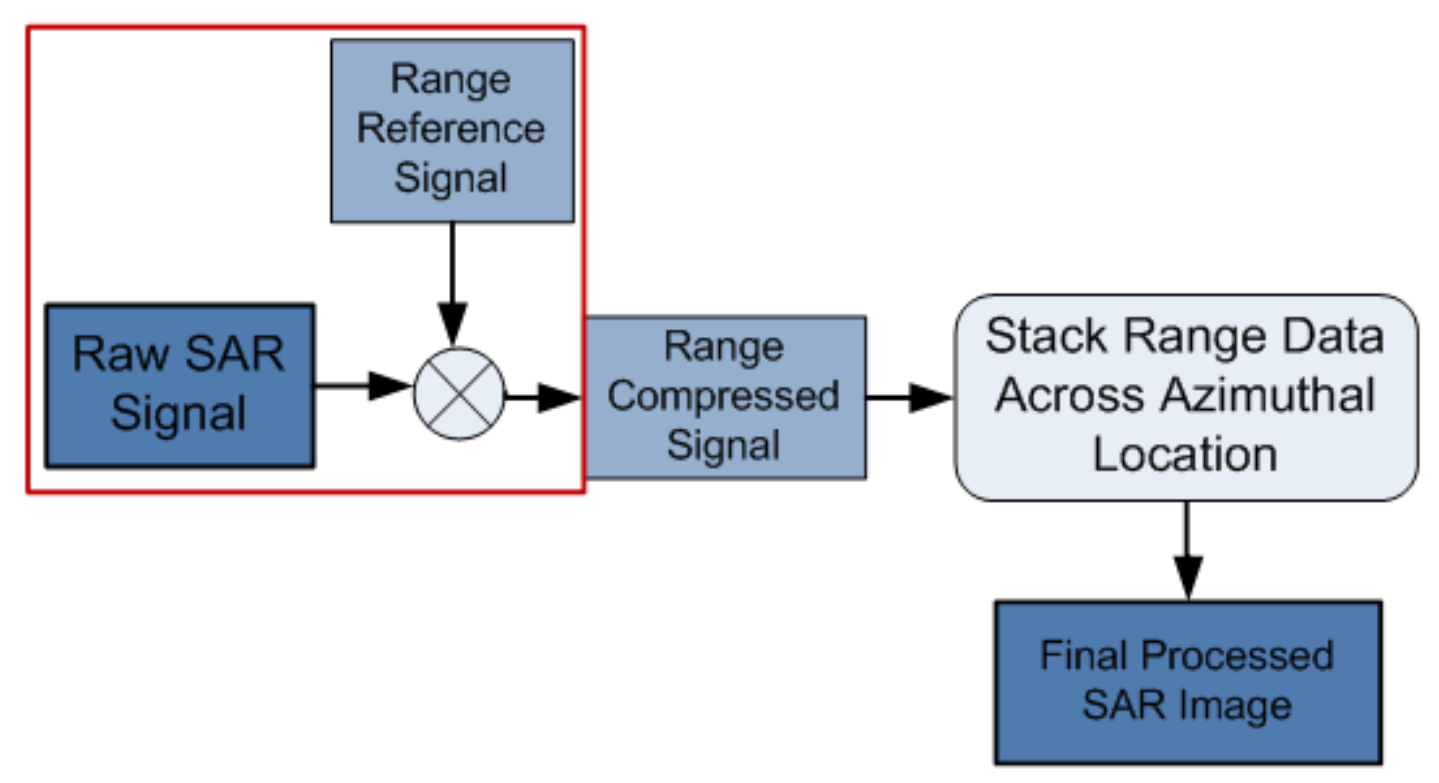

FIGURE 7-1: RANGE COMPRESSION SIGNAL PROCESSING BLOCK DIAGRAM

The red box area in Figure 7-1 accomplishes hardware-based range compression as described in section 6.1 above. Following range compression, the received signal at each location along the rail is sequentially arranged along the azimuthal dimension to produce the final image, see Figure 1-5.

\subsection{RANGE COMPRESSION}

Figure 7-2 below illustrates range compression effects with a matched filtered fast time signal example for one azimuthal location along the rail. 


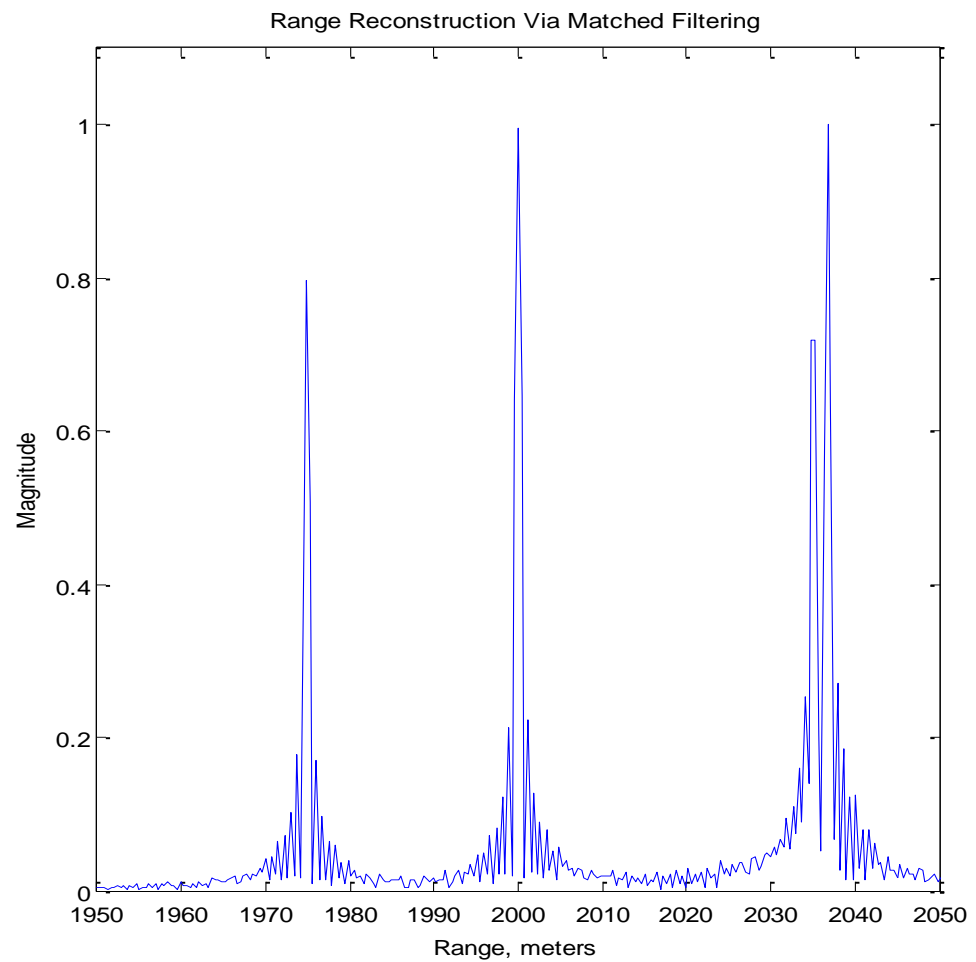

FIGURE 7-2: EXAMPLE RANGE COMPRESSION RESULTS FROM MATCHED FILTERING; RECEIVED SIGNAL MAGNITUDE LEVELS NORMALIZED TO THE SUM OF ALL FREQUENCY COMPONENTS [6]

The range compression via matched filtering example in Figure 7-2 illustrates three clearly defined targets. The radar slant range distance to target, $\mathrm{x}_{\mathrm{n}}$, is related to signal travel time $t_{n}$ to and from the target time by:

$$
t_{n}=\frac{2 x_{n}}{c} \quad n=1,2,3, \ldots
$$

Figure 7-2 has been transformed from time to distance through equation 7-1. After range compression and time to distance translation (equation 7-1), the resulting fast time domain signal contains peaks at specific distances that represent detected targets. 
In real-time SAR imaging, range compression, range cell migration correction, and azimuthal compression are the primary signal processing stages. The radar echo signal return from targets is typically sampled by analog to digital converter (A/D) and sent to the range compression module. Frequency domain range compression involves FFT of sampled chirp echoes, multiplication with the frequency domain reference function, and IFFT. The signal is then stored for range cell migration correction and azimuthal compression.

In this project, only a hardware implementation of the SAR range compression stage is considered. The received target echo is correlated in hardware with the transmitted chirped FM pulse.

\subsubsection{HARDWARE RANGE COMPRESSION}

Let $r(t)$ denote the received chirp signal. Mixing two signals results in multiplication of two time-domain signals which equals the inverse Fourier transform of the convolved (defined with “*”) frequency-domain signals.

$$
s(t)=r(t) P(t)=\mathcal{F}^{-1}[\mathcal{F}(r(t)) * \mathcal{F}(P(t))]
$$

where $\mathrm{s}(\mathrm{t})$ is the pulse compressed chirp signal and $\mathrm{P}(\mathrm{t})$ is the transmitted chirp signal. Since $r(t)$ and $P(t)$ are both chirp signals, the mixing operation results in [6]:

$$
s(t)=r^{*}(t) e^{j \beta t+j \alpha t^{2}}=\sum_{n} A_{n} e^{j \beta t_{n}-j \alpha t_{n}^{2}} e^{j 2 \alpha t_{n} t}
$$

where $\beta$ is the lowest chirp frequency, $\alpha$ is the chirp rate, and $t_{n}$ is the time delay associated with the $\mathrm{n}^{\text {th }}$ target. The summation includes an amplitude term $\mathrm{A}_{\mathrm{n}}$, dependent 
on the target's radar cross section and transmit signal amplitude. The second exponential term $\left(e^{j 2 \alpha t_{n} t}\right)$ in (7-3) defines the range compressed frequency for each object $(n)$ in the target scene. From the summation, $\mathrm{s}(\mathrm{t})$ is the Fourier series of target reflections with distance-dependent frequencies. In Figure 7-3, the Fourier transform is applied to s(t), $S(\omega)$, which results in peaks similar to Figure 7-2.

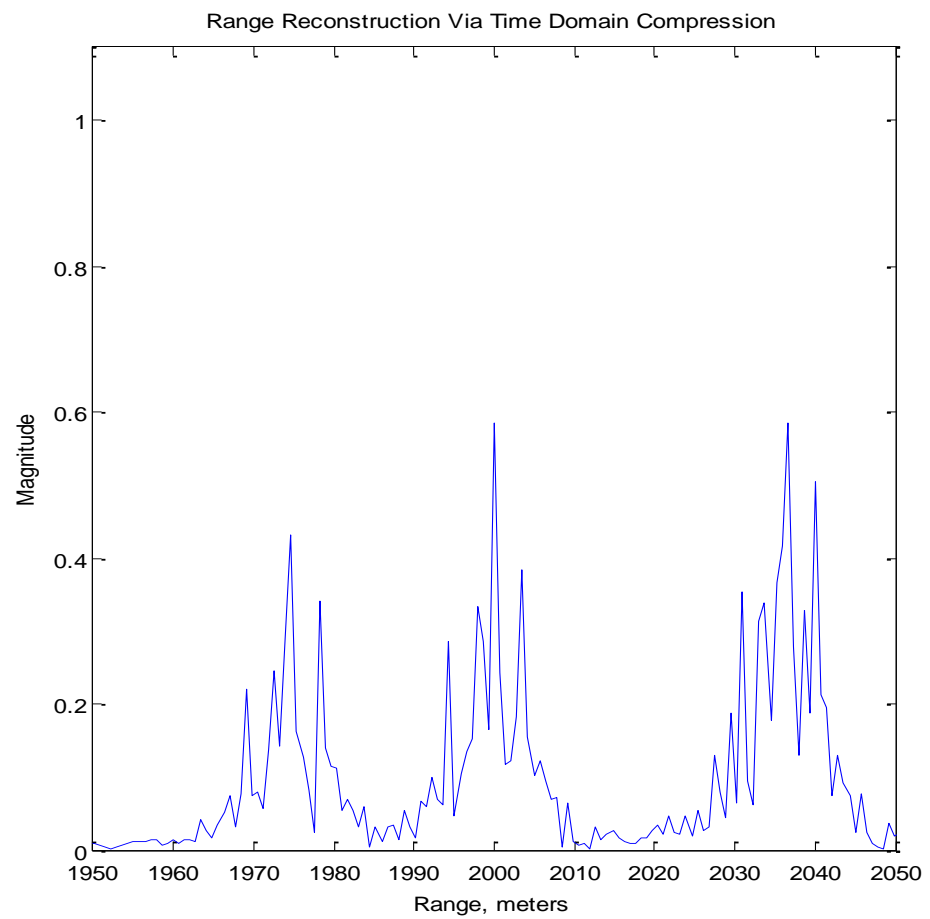

FIGURE 7-3: RANGE COMPRESSION RESULTS FROM PULSE COMPRESSION AND FFT, MAGNITUDE OF RECEIVED SIGNAL LEVELS NORMALIZED TO THE SUM OF ALL FREQUENCY COMPONENTS [6]

Again, the time axis (x-axis) is converted to range distance to create the target scene range measurement using the relation [6]:

$$
x(\text { meters })=\frac{c \omega}{4 \alpha}
$$


where $\mathrm{x}$ is the range distance $(\mathrm{m}), \mathrm{c}$ is the speed of light in vacuum $\left(\sim 3 \mathrm{x} 10^{8} \mathrm{~m} / \mathrm{s}\right), \omega$ is the received signal frequency $(\mathrm{rad} / \mathrm{sec})$, and $\alpha$ is the chirp rate $(\mathrm{Hz} / \mathrm{sec})$.

\subsection{AZIMUTHAL COMPRESSION}

Azimuthal compression is implemented during signal processing to determine a target's physical location in the flight path direction. Without azimuthal compression, a target may appear in multiple fast time data samples across the flight path, even if it should only appear in a single data sample. A matched filtered range compression operation, as described in section 7.1, is applied across the fast time data samples to compress target locations to a narrow range of azimuthal distances in the final image.

To perform successful azimuthal compression, the matched filtering operation requires a well-defined azimuthal reference signal. Unfortunately, project time constraints did not allow sufficient time to define this reference signal and apply it to the matched filtering algorithm. Azimuthal compression algorithm development is a future Cal Poly SAR project. The greatest challenge for future projects is defining the azimuthal reference signal to use for proper azimuthal compression. 
Multiple data sets were collected with targets placed at multiple locations within the radar system's detection region. In order to verify system functionality, single targets were used to create signal reflections from defined target locations. Due to system limitations and the lack of final image azimuthal compression, flat square copper targets are used. As the radar platform traverses the rail, target-reflected RF energy is received primarily when the platform faces the target. Thus, the system's azimuthal resolution is determined by target azimuthal length measurements in the resulting image. Similarly, in the range direction, because targets appear much brighter in the final images compared to ground reflections, the system's range resolution is measured by comparing the target's physical location with those measured in the resulting image.

\subsection{SINGLE TARGET MEASUREMENTS}

In Figure 8-1, a 3'x3' flat copper target is located 10 feet away from the radar system, 5 feet from the edge of the radar rail, and centered in the middle of the rail. 


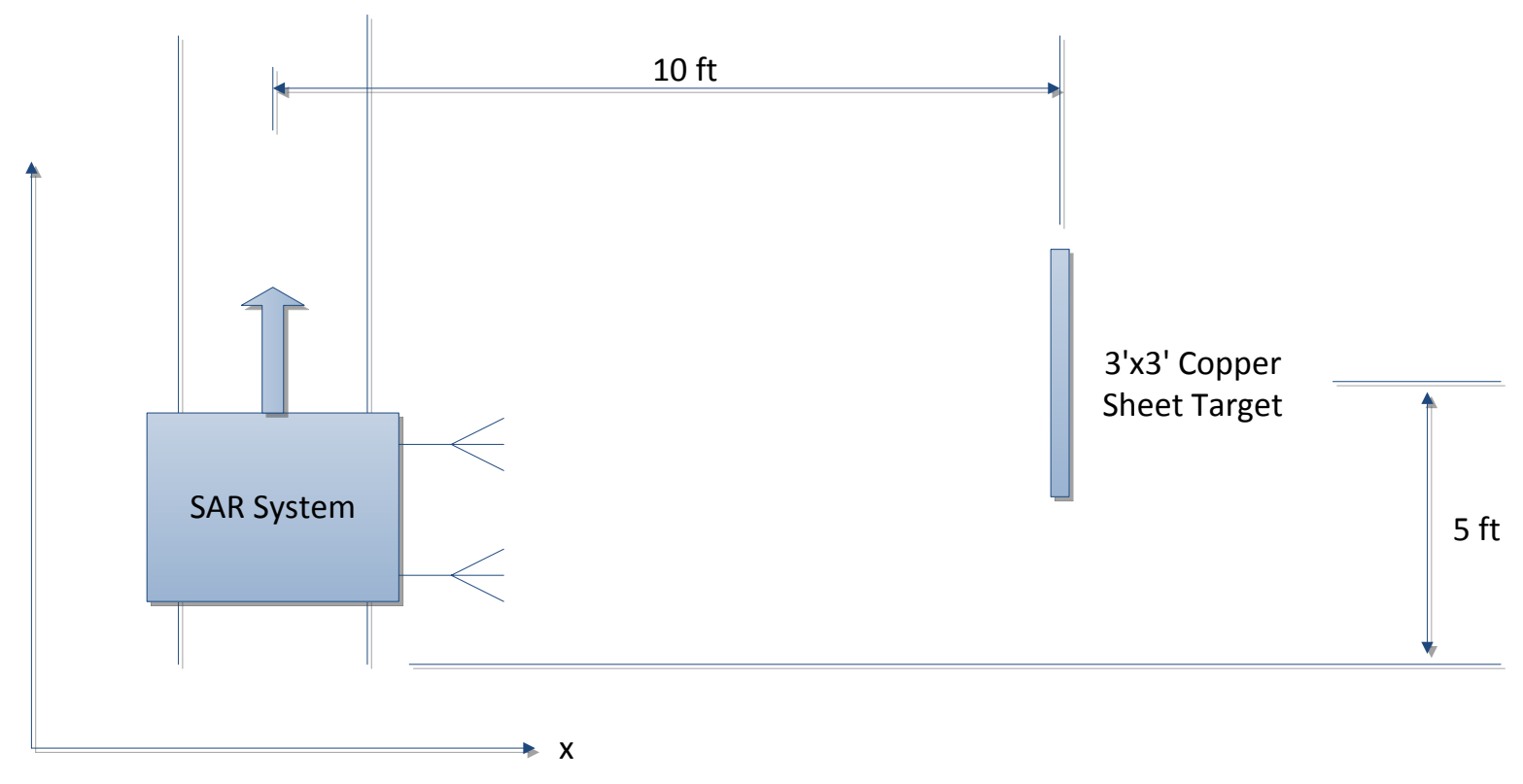

FIGURE 8-1: TARGET SCENE GEOMETRY; SHEET TARGET 10 FT FROM RADAR, 5 FT FROM EDGE OF RAIL MOTION

Within the target scene shown in Figure 8-1, 50 data points were collected at 2.4" rail increments. At the $25^{\text {th }}$ data point, the radar platform is centered in the rail track and directly facing the target. FFT of data collected at the $25^{\text {th }}$ data point is shown in Figure 8-2; three peaks represent possible targets at different ranges. However, in the actual target scene, only one target is present.

The two lowest frequency peaks (Figure 8-2) are caused by antenna to antenna coupling which allows direct signal transmission to the RX antenna without target scene reflections. Figure 8-3 shows FFT data resulting from the radar TX and RX ports connected by 2' coaxial cables. Figure 8-3 clearly shows the two non-ideal target peaks appearing in this non-radiated signal test. Therefore, the minimum radar system range is limited to approximately $5 \mathrm{ft}$. Targets within $5 \mathrm{ft}$ may not be discernible over the FFT range peaks presented by system limitations. 


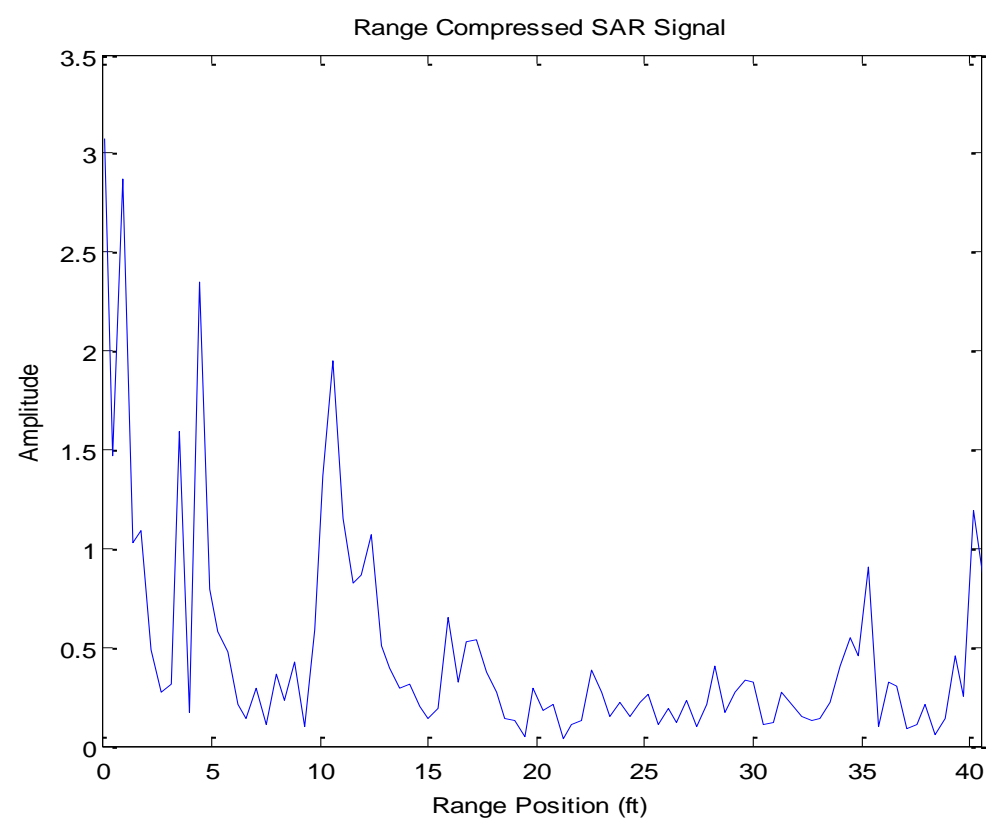

FIGURE 8-2: RAW RANGE DATA COLLECTED 5 FT ACROSS THE RAIL WITH 3X3' TARGET AT 10 FT RANGE DISTANCE

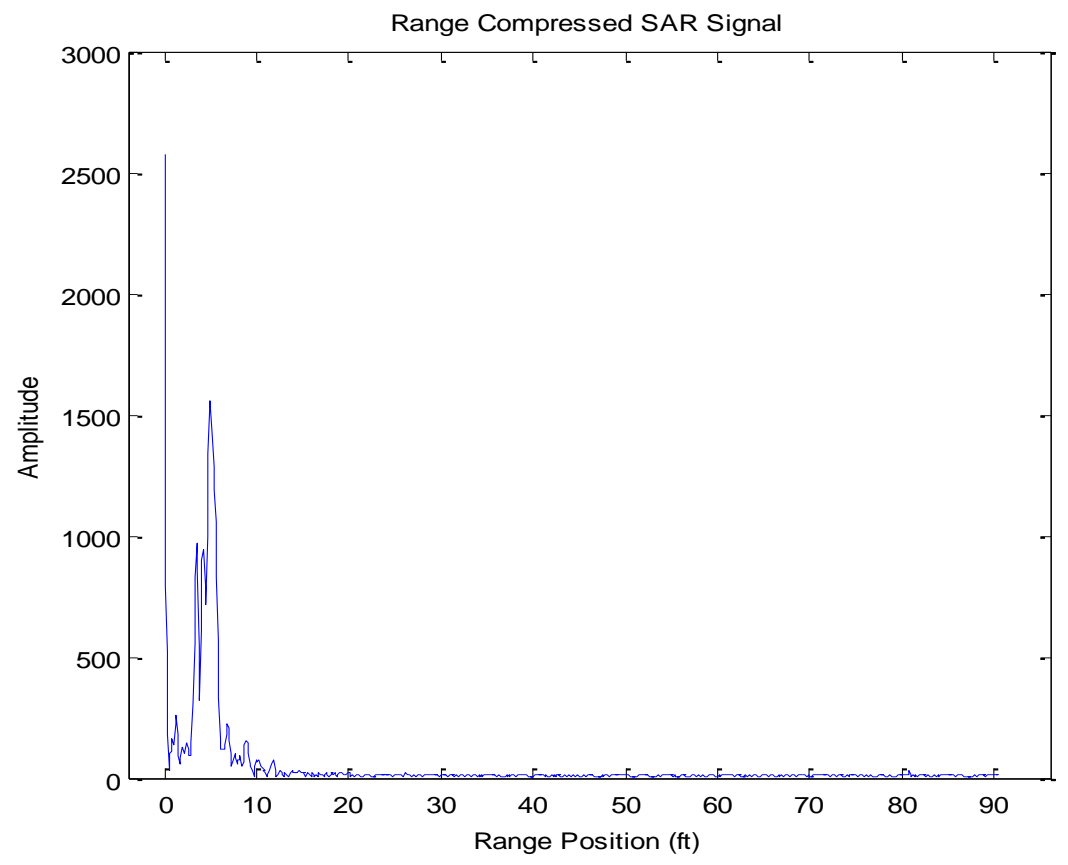

FIGURE 8-3: RANGE DATA CALIBRATION COLLECTED WITH TX AND RX COAX CABLES CONNECTED, ANTENNAS REMOVED 
Antenna coupling calibration improves image clarity. Figure 8-4 shows the image generated from the Figure 8-1 target scene geometry.

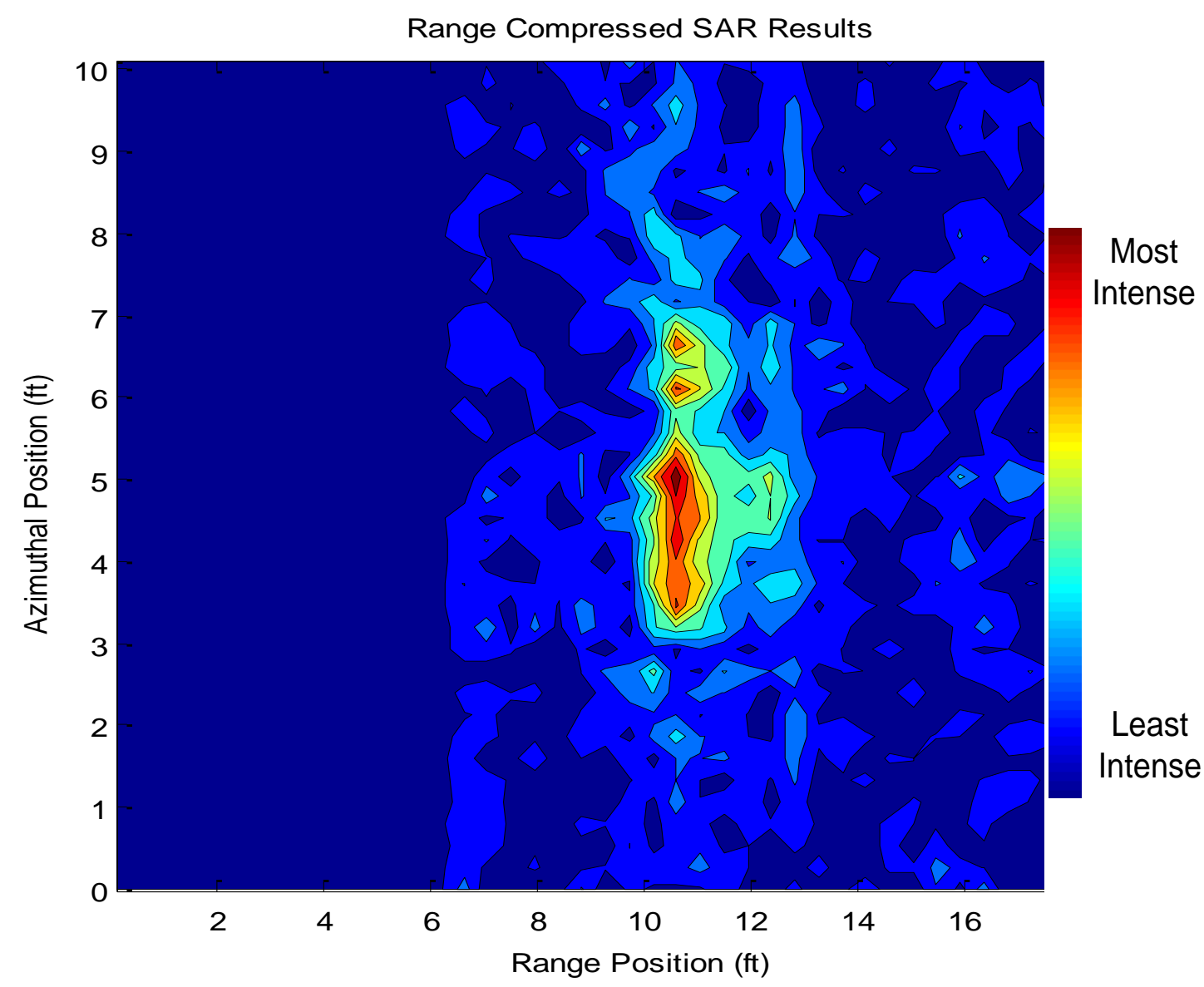

\section{FIGURE 8-4: SINGLE 3'X3' COPPER SHEET TARGET LOCATED 10 FT FROM RADAR}

Figure 8-4 shows received signal intensity across range and azimuthal positions. The dark red color corresponds to maximum amplitude in each FFT azimuthal scan, as in Figure 8-2 above. At 10 feet from the radar platform, the red region includes $1 \mathrm{ft}$ of azimuthal length. Areas around the main target display reduced yet significant signal intensities from potential targets. The yellow area around the red region also includes the desired 3'x3' target. When the radar platform directly faces the target, maximum energy 
is reflected back to the antennas. On either side of the target center, reduced amplitude energy is reflected from the target, hence the less intense yellow color. Green and light blue areas around the target and beyond the target's range can be attributed to clutter from ground reflections. If the radar signal reflects from other surfaces after target reflection, time is added to the signal's propagation time, resulting in the target appearing at a larger range distance in the final image.

In Figure 8-5, a 3'x3' flat copper target is positioned 15 feet away from the radar platform, 2 feet from the radar rail end.

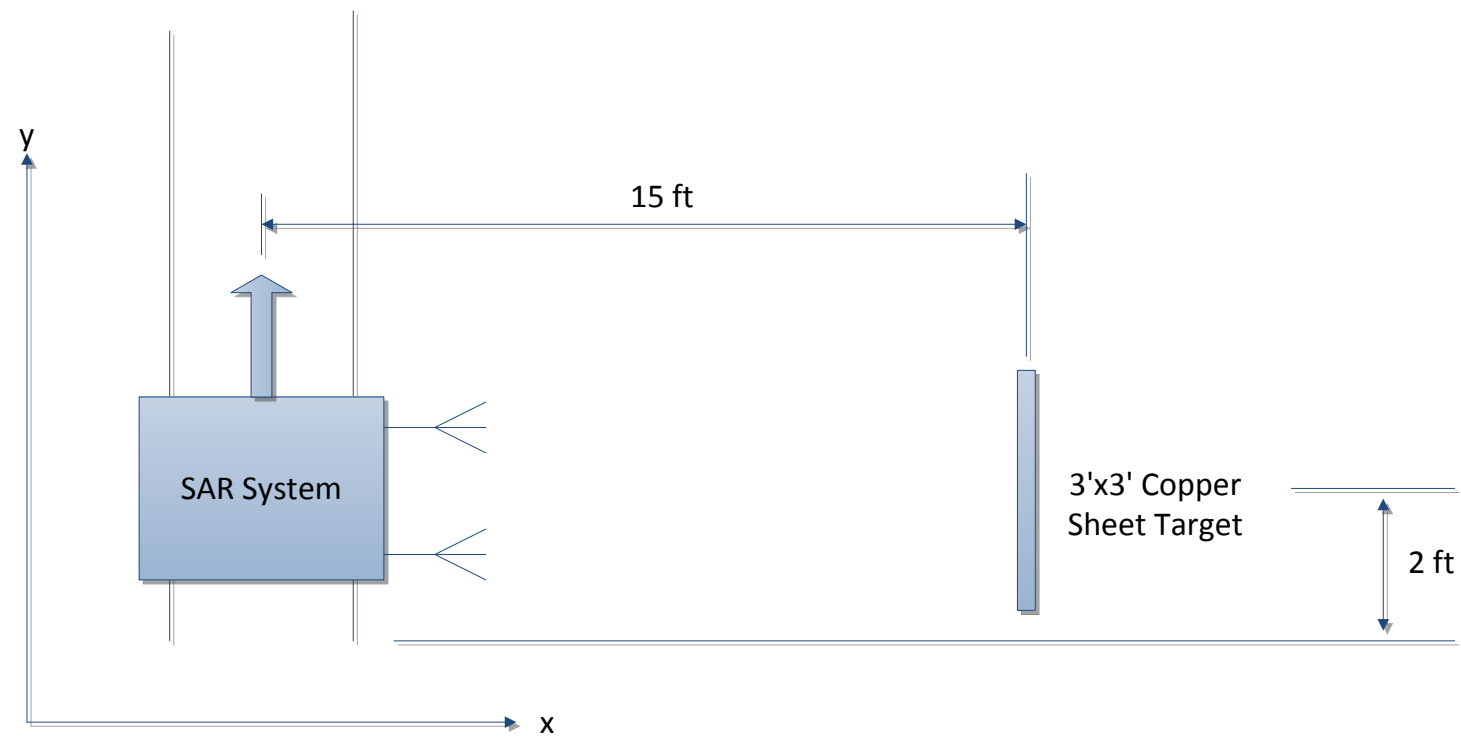

FIGURE 8-5: TARGET SCENE GEOMETRY FOR TARGET LOCATED 15 FT FROM THE RADAR AND 2 FT FROM RAIL LIMIT

For this data set, 39 data points were collected along the rail at 3.07" intervals. The resulting target scene image is shown in Figure 8-6 below. 


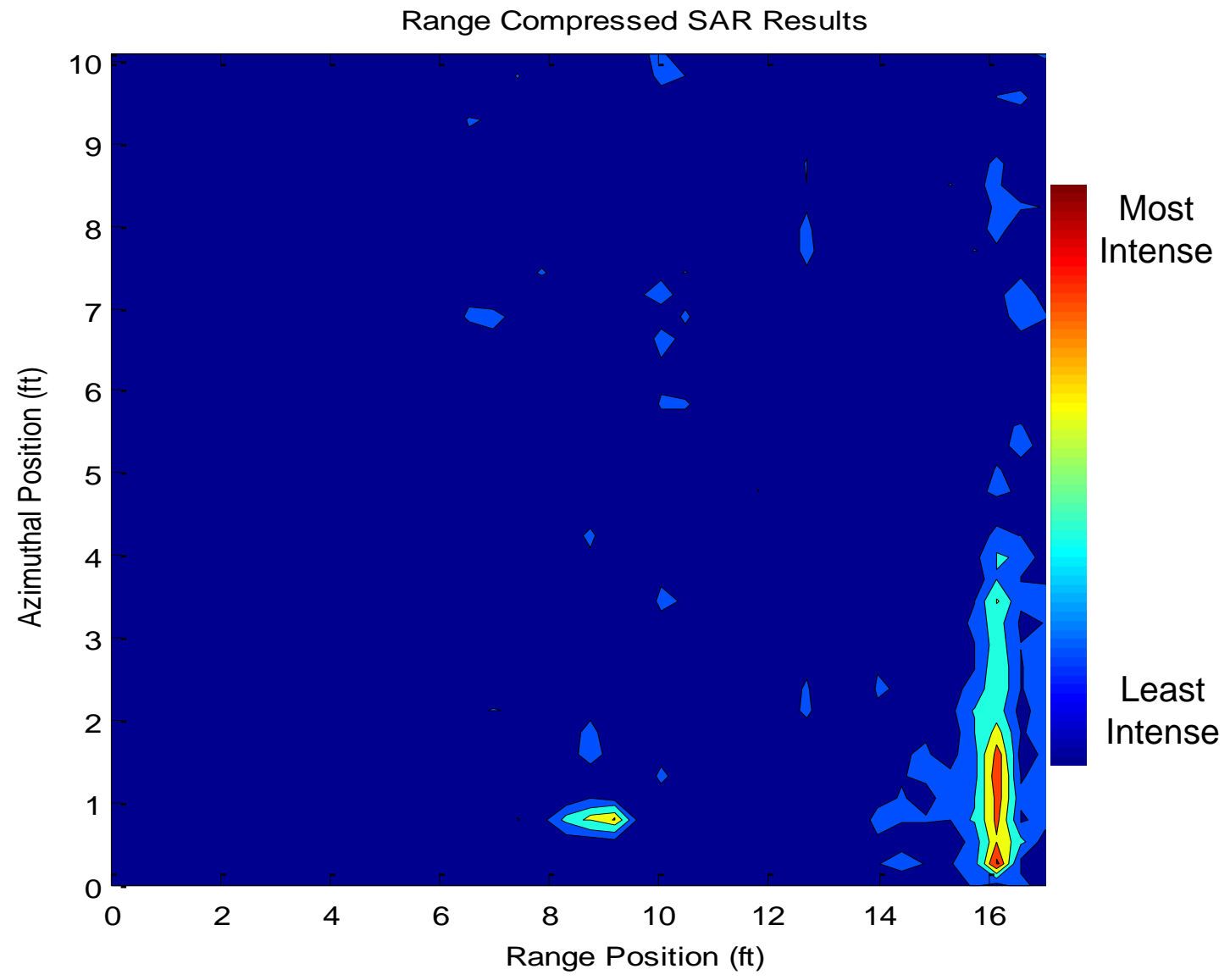

FIGURE 8-6: SINGLE 3'X3' COPPER SHEET TARGET LOCATED 15 FT FROM RADAR

The Figure 8-6 image shows the target location in red, with surrounding artifacts and smaller amplitude reflections. Even without azimuthal compression, the target appears to have an azimuthal width less than the actual target width. Target narrowing in the image without azimuthal compression techniques may be caused by a slightly angled target, which reduces the target's effective radar cross-section resulting in fewer azimuthal target reflection data samples received by the radar. This is illustrated by the long lightblue area at a range of 16 feet and an azimuthal location span of 2 to 5 feet. This lightblue area may be due to target rotation away from the rail center and continued reflected signal reception after the platform passed directly in front of the target. Though the 
target appears at $16 \mathrm{ft}$ range, the additional $1 \mathrm{ft}$ range is associated with the time delay through the RF front-end in the TX and RX directions. In this data set, the extra time delay was not calibrated out during data collection. Similar to the discussion surrounding Figure 8-3 above, a through-line connection between the TX and RX front-ends with the antennas removed would have allowed for the additional time delay to be compensated in processing. Because data was collected over many weeks, the system was rebuilt multiple times with the goal of achieving better results. This required the calibration procedure to be repeated each time the system was re-built, however, this data set was collected without calibration information.

Overall, these two data sets and corresponding images prove that this system functions with reliable range accuracy. However, azimuthal compression omission leads to unexpected images for rail-oblique (angled) targets. 
This thesis presents a scaled synthetic aperture radar system designed for close range $(<100 \mathrm{ft})$ imaging. Sub-systems described in this thesis include the antenna, RF signal chain, radar movement, LabView, and signal processing. A Vivaldi flare antenna pair was designed, fabricated and tested. This antenna pair was connected to a frequency chirp (1.2 to $2.5 \mathrm{GHz}$ ) transceiver. The LabView sub-system controls data collection as well as antenna and RF sub-system movement along a $10 \mathrm{ft}$ guide rail. The movement subsystem (rail and moving platform) was built using supplies available at home improvement stores. After a full sweep across the rail, signal processing algorithms similar to the Range Doppler Algorithm generate a two-dimensional SAR image of the target scene. Hardware range compression images are presented to summarize system capabilities. This thesis describes major considerations in scaled SAR system development. The thesis by Jason Schray completes the scaled SAR imaging system description.

\subsection{FUTURE PLANS}

Many features were omitted from this system due to time constraints. Future projects may experiment with greater RF power output stage amplifiers to increase measured range swaths. The sub-system with the greatest improvement potential is signal processing. Implementing successful azimuthal compression and range cell migration algorithms will significantly improve image quality. However, signal processing improvements depend on experimentation with the number of azimuthal points recorded along the rail. Finally, 
condensing the entire RF and data collection system into a small package would allow remote UAV operation in conjunction with the Cal Poly Aerospace Engineering department. 


\section{REFERENCES}

[1] G. L. Charvat, “A Low-Power Radar Imaging System,” Ph.D. dissertation, Dept. of Electrical and Computer Engineering, Michigan State University, East Lansing, MI, 2007.

[2] B. Panzer, "Development of an Electrically Small Vivaldi Antenna: The CReSIS Aerial Vivaldi (CAV-A),” CReSIS Technical Report, no. 135, pp. 81, 12/2007.

[3] D.H. Schaubert et al., "Endfire tapered slot antennas on dielectric substrates," IEEE Trans. Antennas and Propagation, vol.33, no.12, pp.1392,1400, Dec 1985

[4] J. Shin and D.H. Schaubert, "A parameter study of stripline-fed Vivaldi notch-antenna arrays," IEEE Trans. Antennas and Propagation, vol.47, no.5, pp.879,886, May 1999

[5] M. Kobayashi and N. Sawada, "Analysis and synthesis of tapered microstrip transmission lines," in IEEE Trans. Microw. Theory Tech., vol.40, no.8, pp.16421646, Aug 1992

[6] M. Soumekh. Synthetic aperture radar signal processing with MATLAB algorithms. New York: J. Wiley, 1999

[7] W. Ong (2005). "Commercial off the Shelf Direct Digital Synthesizers for Digital Array Radar,” Master's Thesis, Naval Postgraduate School.

[8] A.A. Ksienski and R.B. Mcghee, "Radar signal processing for angular resolution beyond the rayleigh limit," Radio and Electronic Engineer, vol.34, no.3, pp.161,174, September 1967

[9] I.G. Cumming et al., "Processing of Azimuth-Invariant Bistatic SAR Data Using the Range Doppler Algorithm," IEEE Trans. Geosci. Remote Sens., vol.46, no.1, pp.14,21, Jan. 2008

[10] Antenna Introduction / Basics. (2007). [Online]. Available: http://www.phys.hawaii.edu/ anita/new/

[11] Acfr.usyd.edu.au, 'Sensor systems (KC-2) - ACFR - The University of Sydney', 2015. [Online]. Available: http://www.acfr.usyd.edu.au/education/sensorSystems.shtml.

[12] Y. Erdogan (2009). "Parametric study and design of Vivaldi antennas and arrays," Master's Thesis, Middle East Technical University.

[13] R.L. Smith (2002). "Micro synthetic aperture radar using FW/CW technology," Master's Thesis, Brigham Young University.

[14] M.C. Edwards (2009). "Design of continuous-wave synthetic aperture radar system with analog dechirp," Master's Thesis, Brigham Young University. 
[15] M. Budge (2011). EE 710 - ST: Radar Waveforms \& Signal Processing. Lecture conducted from University of Alabama Huntsville.

[16] S. Orfanidis (2008). Antenna Arrays. In Electromagnetic Waves and Antennas, pp. 909-936, ECE Department Rutgers University.

[17] D. Mandeep and M. Nicholas, 'Design An X-Band Vivaldi Antenna', Mwrf.com, 2015. [Online]. Available: http://mwrf.com/markets/design-X-band-vivaldiantenna.

[18] M.L. Boas (2006). Mathematical Methods in the Physical Sciences.Wiley, pp. 839, ISBN 978-0-471-19826-0.

[19] I.G. Cumming and F.H. Wong (2005). Digital processing of synthetic aperture radar data: Algorithms and implementation. Boston: Artech House.

[20] Y. Chan and V. Koo, 'AN INTRODUCTION TO SYNTHETIC APERTURE RADAR (SAR)', Progress In Electromagnetics Research B, vol. 2, pp. 27-60, 2008. 